TRANSACTIONS OF THE

AMERICAN MATHEMATICAL SOCIETY

Volume 350, Number 8, August 1998, Pages 3321-3339

S 0002-9947(98)02117-5

\title{
LIE IDEALS IN TRIANGULAR OPERATOR ALGEBRAS
}

\author{
T. D. HUDSON, L. W. MARCOUX, AND A. R. SOUROUR
}

\begin{abstract}
We study Lie ideals in two classes of triangular operator algebras: nest algebras and triangular UHF algebras. Our main results show that if $\mathfrak{L}$ is a closed Lie ideal of the triangular operator algebra $\mathcal{A}$, then there exist a closed associative ideal $\mathcal{K}$ and a closed subalgebra $\mathfrak{D}_{\mathcal{K}}$ of the diagonal $\mathcal{A} \cap \mathcal{A}^{*}$ so that $\mathcal{K} \subseteq \mathfrak{L} \subseteq \mathcal{K}+\mathfrak{D}_{\mathcal{K}}$.
\end{abstract}

\section{INTRODUCTION}

Let $\mathbb{A}$ be an associative complex algebra. Under the Lie multiplication $[x, y]=$ $x y-y x, \mathbb{A}$ becomes a Lie algebra. A Lie ideal in $\mathbb{A}$ is a linear manifold $\mathfrak{L}$ in $\mathbb{A}$ for which $[a, k] \in \mathfrak{L}$ for every $a \in \mathbb{A}$ and $k \in \mathfrak{L}$. In many instances, there is a close connection between the Lie ideal structure and the associative ideal structure of $\mathbb{A}$. This connection has been investigated for prime rings in [6], in [3] for $\mathcal{B}(\mathfrak{H})$ - the set of bounded operators on a Hilbert space $\mathfrak{H}$, and in [10] for certain von Neumann algebras. (See also $[9,4,11]$.) In this paper we pursue this line of investigation for two classes of triangular operator algebras, namely nest algebras and triangular UHF algebras.

The authors would like to thank Frank A. Zorzitto for many helpful conversations.

\section{WEAKLY CLOSED LIE IDEALS IN NEST ALGEBRAS}

Recall that a nest $\mathcal{N}$ on a Hilbert space $\mathfrak{H}$ is a chain of closed subspaces of $\mathfrak{H}$ which is closed under the operations of arbitrary intersections and closed linear spans, and which includes $\{0\}$ and $\mathfrak{H}$. The nest algebra $\mathcal{T}(\mathcal{N})$ is the algebra of all operators on $\mathfrak{H}$ leaving every member of $\mathcal{N}$ invariant. This is always closed in the weak operator topology. The diagonal $\mathfrak{D}(\mathcal{N})$ of a nest algebra $\mathcal{T}(\mathcal{N})$ is the von Neumann subalgebra $\mathcal{T}(\mathcal{N}) \cap \mathcal{T}(\mathcal{N})^{*}$. If $E, F \in \mathcal{N}$ with $E<F$, then $F-E$ is called an interval of the nest. The nonzero minimal intervals are called atoms. A nest is atomic if the atoms of the nest span $\mathfrak{H}$. We refer the reader to [1] for more information on nest algebras.

Our main result, Theorem 12 , shows that for every weakly closed Lie ideal $\mathfrak{L}$ in $\mathcal{T}(\mathcal{N})$, there exist a corresponding weakly closed associative ideal $\mathcal{K}$ and a von Neumann subalgebra $\mathfrak{D}_{\mathcal{K}}$ of $\mathfrak{D}(\mathcal{N})$ such that

$$
\mathcal{K} \subseteq \mathfrak{L} \subseteq \mathcal{K}+\mathfrak{D}_{\mathcal{K}}
$$

Received by the editors October 4, 1996.

1991 Mathematics Subject Classification. Primary 47D25, 46K50.

This research was supported in part by an NSF grant (to Hudson) and by NSERC (of Canada) grants (to Marcoux and Sourour). 
It may be instructive to illustrate our result in the simplest finite dimensional case. This particular example may be known, but we have been unable to discover a reference for it.

The set $\mathbf{T}_{n}$ of $n \times n$ upper triangular matrices is an example of a nest algebra. An elementary calculation shows that there is a bijective correspondence between the set of associative ideals in $\mathbf{T}_{n}$ and the set of non-decreasing functions $f:\{0,1, \ldots, n\} \rightarrow\{0,1, \ldots, n\}$ satisfying $f(j) \leq j$ for all $0 \leq j \leq n$. This correspondence is given by $f \leftrightarrow I_{f}:=\left\{\left[a_{i j}\right] \in \mathbf{T}_{n} \mid a_{i j}=0\right.$ if $\left.i>f(j)\right\}$. We may associate to each associative ideal $I$ of $\mathbf{T}_{n}$ a subalgebra $\mathfrak{D}_{I}$ of the diagonal $\mathfrak{D}_{n}$, namely

$\mathfrak{D}_{I}=\left\{\operatorname{diag}\left(d_{1}, \ldots, d_{n}\right) \in \mathfrak{D}_{n} \mid d_{k}=d_{k+1}\right.$ if $\left.a_{k, k+1}=0 \forall\left[a_{i j}\right] \in I, \forall 1 \leq k \leq n-1\right\}$.

Theorem 1. If $\mathfrak{L}$ is a Lie ideal of $\mathbf{T}_{n}$, then the intersection $\mathcal{K}$ of $\mathfrak{L}$ with the space of strictly upper triangular matrices is an associative ideal of $\mathbf{T}_{n}$ satisfying $\mathcal{K} \subseteq \mathfrak{L} \subseteq \mathcal{K}+\mathfrak{D}_{\mathcal{K}}$

It is worth pointing out that in this example, the converse also holds, namely: if $\mathcal{K}$ is any associative ideal of $\mathbf{T}_{n}$, and $\mathfrak{L}$ is any linear manifold satisfying $\mathcal{K} \subseteq \mathfrak{L} \subseteq$ $\mathcal{K}+\mathfrak{D}_{\mathcal{K}}$, then $\mathfrak{L}$ is a Lie ideal of $\mathbf{T}_{n}$.

For the general case, we shall need the following characterization of the weakly closed associative ideals of nest algebras as obtained in [2].

Theorem 2 (Erdos-Power). If $\mathcal{I}$ is a weakly closed ideal of $\mathcal{T}(\mathcal{N})$, then $\mathcal{I}$ has the form

$$
\mathcal{I}=\{X \in \mathcal{B}(\mathfrak{H}) \mid(I-\widetilde{E}) X E=0 \quad \forall E \in \mathcal{N}\},
$$

where $E \mapsto \widetilde{E}$ is a left order continuous order homomorphism of $\mathcal{N}$ into $\mathcal{N}$ such that $\widetilde{E} \leq E$ for each $E \in \mathcal{N}$.

Throughout this section, let $\widetilde{E}$ denote the image of $E$ under the order homomorphism of $\mathcal{N}$ into itself corresponding to the weakly closed ideal $\mathcal{I}$.

The following lemma, while elementary, will also prove useful.

Lemma 3. Let $\mathcal{I}$ be a weakly closed ideal of $\mathcal{T}(\mathcal{N})$ and $X \in \mathcal{T}(\mathcal{N})$. Then

$$
\mathcal{I}=\{X \in \mathcal{T}(\mathcal{N}) \mid(E-\widetilde{E}) X(E-\widetilde{E})=0 \text { for all } E \in \mathcal{N}\}
$$

Proof. First assume that $(E-\widetilde{E}) X(E-\widetilde{E})=0$ for every $E \in \mathcal{N}$. Let $E \in \mathcal{N}$. Since $\widetilde{E} \leq E$ and $X E=E X E$, we have

$$
\begin{aligned}
(I-\widetilde{E}) X E & =(I-\widetilde{E}) E X E-(I-\widetilde{E}) E X \widetilde{E} \\
& =(I-\widetilde{E}) E X(E-\widetilde{E}) \\
& =(E-\widetilde{E}) X(E-\widetilde{E})=0 .
\end{aligned}
$$

Since $E \in \mathcal{N}$ was arbitrary, by Theorem 2 it follows that $X \in \mathcal{I}$.

Conversely, if $X \in \mathcal{I}$, then $0=(I-\widetilde{E}) X E=(E-\widetilde{E}) X(E-\widetilde{E})$ for all $E \in \mathcal{N}$.

Let $\mathfrak{L}$ be a Lie ideal of $\mathcal{T}(\mathcal{N})$, (weakly) closed in the weak operator topology. Define

$$
\mathcal{K}:=\overline{\operatorname{span}}^{w}\{P T(I-P) \mid T \in \mathfrak{L}, P \in \mathcal{N}\},
$$

where $\bar{S}^{w}$ denotes the weak operator topology closure of $S, S \subseteq \mathcal{B}(\mathfrak{H})$.

We now show that $\mathcal{K}(=\mathcal{K}(\mathfrak{L}))$ is in fact a weakly closed associative ideal of $\mathcal{T}(\mathcal{N})$ satisfying $\mathcal{K} \subseteq \mathfrak{L} \subseteq \mathcal{K}+\mathfrak{D}(\mathcal{N})$ 
Lemma 4. Let $\mathfrak{L}$ be a weakly closed Lie ideal in $\mathcal{T}(\mathcal{N})$, and let $\mathcal{K}$ be defined as above. Then

$$
\mathcal{K}=\overline{\operatorname{span}}^{w}\{T \in \mathfrak{L} \mid \exists P \in \mathcal{N} \text { with } T=P T(I-P)\} .
$$

Proof. We will show that $G_{1}=G_{2}$, where $G_{1}:=\{P T(I-P) \mid T \in \mathfrak{L}, P \in \mathcal{N}\}$ and $G_{2}:=\{T \in \mathfrak{L} \mid \exists P \in \mathcal{N}$ with $T=P T(I-P)\}$. It is obvious that $G_{2} \subseteq G_{1}$. If $P T(I-P) \in G_{1}$, where $T \in \mathfrak{L}$, then since $T \in \mathcal{T}(\mathcal{N})$, we have $P T(I-P)=$ $P T-P T P=P T-T P$. Thus, $P T(I-P) \in \mathfrak{L}$, and since $P(P T(I-P))(I-P)=$ $P T(I-P) \in G_{2}, G_{1} \subseteq G_{2}$.

Proposition 5. Let $\mathfrak{L}$ be a weakly closed Lie ideal of $\mathcal{T}(\mathcal{N})$ and $\mathcal{K}$ be as defined above. Then $\mathcal{K}$ is a weakly closed associative ideal of $\mathcal{T}(\mathcal{N})$ contained in $\mathfrak{L}$.

Proof. Clearly $\mathcal{K}$ is a weakly closed subspace of $\mathcal{T}(\mathcal{N})$. Lemma 4 proves that $\mathcal{K} \subseteq \mathfrak{L}$. Let $A \in \mathcal{T}(\mathcal{N})$. If $X \in \mathfrak{L}$ and $X=P X(I-P)$ for some $P \in \mathcal{N}$, then

$$
[P A P, X]=(P A P) X-P X(I-P)(P A P)=A P X=A X \text {. }
$$

Since $X \in \mathfrak{L}$ and $P A P \in \mathcal{T}(\mathcal{N})$, we have $A X=[P A P, X] \in \mathfrak{L}$. Moreover,

$$
P A X(I-P)=P A P X(I-P)=A P X(I-P)=A X,
$$

so $A X \in \mathcal{K}$. Since $\mathcal{K}$ is closed under weak spans, we see that $\mathcal{K}$ is a weakly closed left ideal of $\mathcal{T}(\mathcal{N})$. Similarly, by considering $[X,(I-P) A(I-P)]$, we conclude that $\mathcal{K}$ is a right ideal of $\mathcal{T}(\mathcal{N})$.

Throughout the paper, we use $\mathcal{K}$ to denote the weakly closed associative ideal corresponding to the weakly closed Lie ideal $\mathfrak{L}$, as constructed above. We now require a couple of technical lemmas.

Lemma 6. Let $\mathfrak{L}$ be a weakly closed Lie ideal of $\mathcal{T}(\mathcal{N})$ and $\mathcal{K}$ the corresponding associative ideal. Suppose that $E, F \in \mathcal{N}$ with $\widetilde{E}<F<E$. If $[F-\widetilde{E}, X] \in \mathcal{K}$ for some $X \in \mathcal{T}(\mathcal{N})$, then $(F-\widetilde{E}) X(E-F)=0$.

Proof. Since $[F-\widetilde{E}, X] \in \mathcal{K}$, it follows from Theorem 2 that $(I-\widetilde{E})[F-\widetilde{E}, X] E=0$. Since $(F-\widetilde{E})(E-F)=0$,

$$
\begin{aligned}
(F-\widetilde{E}) X(E-F) & =(F-\widetilde{E}) X(E-F)-(F-\widetilde{E}) X(F-\widetilde{E})(E-F) \\
& =(F-\widetilde{E})[F-\widetilde{E}, X](E-F) \\
& =(F-\widetilde{E})(I-\widetilde{E})[F-\widetilde{E}, X] E(E-F)=0,
\end{aligned}
$$

as desired.

For a nest algebra $\mathcal{T}(\mathcal{N})$, there is an expectation (generally not unique) from $\mathcal{T}(\mathcal{N})$ onto the diagonal $\mathfrak{D}(\mathcal{N})$ (see $[1$, p. 90]).

Lemma 7. Let $\mathfrak{L}$ be a weakly closed Lie ideal of $\mathcal{T}(\mathcal{N})$ and $\mathcal{K}$ the corresponding associative ideal. Let $\pi: \mathcal{T}(\mathcal{N}) \rightarrow \mathfrak{D}(\mathcal{N})$ be an expectation onto $\mathfrak{D}(\mathcal{N}), E \in \mathcal{N}$, and $X \in \mathcal{T}(\mathcal{N})$. If $[F-\widetilde{E}, X-\pi(X)] \in \mathcal{K}$ for every $\widetilde{E}<F<E, F \in \mathcal{N}$, then $(E-\widetilde{E})(X-\pi(X))(E-\widetilde{E})=0$.

Proof. Let $P=E-\widetilde{E}$ and $X_{\pi}=X-\pi(X)$. Let $M(F)=F P X_{\pi} P(I-F)$ for $F \in \mathcal{N}$. If $F<\widetilde{E}$, then $M(F)=(F-F) X_{\pi} P(I-F)=0$, and similarly, if $F>E$, we have $M(F)=(E-\widetilde{E}) X_{\pi}((E-\widetilde{E})-(E-\widetilde{E}))=0$. If either $F=\widetilde{E}$ 
or $F=E$, then clearly $M(F)=0$. If $\widetilde{E}<F<E$, then by Lemma 6 we have $M(F)=(F-\widetilde{E}) X_{\pi}(E-F)=0$.

It now follows that $P X_{\pi} P \in \mathcal{T}(\mathcal{N})^{*}$. But since $P X_{\pi} P$ also belongs to $\mathcal{T}(\mathcal{N})$, we have $P X_{\pi} P \in \mathfrak{D}(\mathcal{N})$. Since $\pi$ is an expectation onto $\mathfrak{D}(\mathcal{N})$, we see that

$$
\begin{aligned}
P X_{\pi} P=\pi\left(P X_{\pi} P\right) & =P \pi(X-\pi(X))) P \\
& =P(\pi(X)-\pi(\pi(X))) P \\
& =P(\pi(X)-\pi(X)) P=0,
\end{aligned}
$$

as desired.

Proposition 8. Let $\mathfrak{L}$ be a weakly closed Lie ideal of $\mathcal{T}(\mathcal{N})$ and $\mathcal{K}$ the corresponding associative ideal. Then $\mathfrak{L} \subseteq \mathcal{K}+\mathfrak{D}(\mathcal{N})$.

Proof. Suppose that $K \in \mathfrak{L}$. Let $\pi$ be any expectation onto $\mathfrak{D}(\mathcal{N})$. We show that $K_{\pi}:=K-\pi(K)$ belongs to $\mathcal{K}$, so that $K=K_{\pi}+\pi(K) \in \mathcal{K}+\mathfrak{D}(\mathcal{N})$.

Fix $E \in \mathcal{N}$. Let $F \in \mathcal{N}$ with $\widetilde{E}<F<E$, and set $R_{F}=F-\widetilde{E}$ and $Y_{F}=$ $\left[R_{F}, K_{\pi}\right]$. Since $\pi(K) \in \mathcal{N}^{\prime}, Y_{F}=\left[R_{F}, K\right]-\left[R_{F}, \pi(K)\right]=\left[R_{F}, K\right] \in \mathfrak{L}$.

We now show that $Y_{F} \in \mathcal{K}$. Since

$$
(F-\widetilde{E}) K F=(F-\widetilde{E}) K(F-\widetilde{E})=(I-\widetilde{E}) K(F-\widetilde{E}),
$$

then

$$
\begin{aligned}
{\left[R_{F}, K\right] } & =(F-\widetilde{E}) K-K(F-\widetilde{E}) \\
& =(F-\widetilde{E}) K(I-F)-\widetilde{E} K(F-\widetilde{E})+(F-\widetilde{E}) K F-(I-\widetilde{E}) K(F-\widetilde{E}) \\
& =(F-\widetilde{E}) K(I-F)-\widetilde{E} K(F-\widetilde{E})
\end{aligned}
$$

We now show that each of $(F-\widetilde{E}) K(I-F)$ and $\widetilde{E} K(F-\widetilde{E})$ lie in $\mathcal{K}$, so that $\left[R_{F}, K\right] \in \mathcal{K}$. Recall that since $K \in \mathfrak{L}$, we have $\left[R_{F}, K\right] \in \mathfrak{L}$. By (1), we see that $\left[\widetilde{E},\left[R_{F}, K\right]\right]=-\widetilde{E} K(F-\widetilde{E})$. But since $\left[R_{F}, K\right]$ belongs to $\mathfrak{L}$, we also have $\left[\widetilde{E},\left[R_{F}, K\right]\right] \in \mathfrak{L}$. But

$$
\begin{aligned}
\widetilde{E}\left[\widetilde{E},\left[R_{F}, K\right]\right](I-\widetilde{E}) & =\widetilde{E}(-\widetilde{E} K(F-\widetilde{E}))(I-\widetilde{E}) \\
& =-\widetilde{E} K(F-\widetilde{E}) \\
& =\left[\widetilde{E},\left[R_{F}, K\right]\right]
\end{aligned}
$$

and so $\left[\widetilde{E},\left[R_{F}, K\right]\right] \in \mathcal{K}$. Similarly, again by $(1),\left[F,\left[R_{F}, K\right]\right]=(F-\widetilde{E}) K(I-F) \in$ $\mathfrak{L}$, and so

$$
\begin{aligned}
F\left[F,\left[R_{F}, K\right]\right](I-F) & =F((F-\widetilde{E}) K(I-F))(I-F) \\
& =(F-\widetilde{E}) K(I-F) \\
& =\left[F,\left[R_{F}, K\right]\right] .
\end{aligned}
$$

It follows that $\left[F,\left[R_{F}, K\right]\right] \in \mathcal{K}$. Hence, $Y_{F}=\left[R_{F}, K\right]=\left[F,\left[R_{F}, K\right]\right]+\left[\widetilde{E},\left[R_{F}, K\right]\right]$ $\in \mathcal{K}$.

Thus, for any $F \in \mathcal{N}$ satisfying $\widetilde{E}<F<E$, we have shown that

$$
[F-\widetilde{E}, K-\pi(K)] \in \mathcal{K} .
$$

So by Lemma 7 , we have $(E-\widetilde{E}) K_{\pi}(E-\widetilde{E})=0$. As $E \in \mathcal{N}$ was arbitrary, by Lemma $3, K_{\pi} \in \mathcal{K}$. 
Recall that, given a weakly closed ideal $\mathcal{I}$ of $\mathcal{T}(\mathcal{N}), \widetilde{E}$ denotes the image of $E \in \mathcal{N}$ under the order homomorphism corresponding to $\mathcal{I}$ given by Theorem 2.

Definition. Let $\mathcal{I}$ be a weakly closed associative ideal of $\mathcal{T}(\mathcal{N})$. We define

$$
\mathfrak{D}_{\mathcal{I}}=\left\{D \in \mathfrak{D}(\mathcal{N}) \mid \text { for all } E \in \mathcal{N} \text { satisfying } \widetilde{E}<E_{-}\right. \text {we have }
$$

$$
\left.(E-\widetilde{E}) D(E-\widetilde{E})=\lambda_{E}(E-\widetilde{E}) \text { for some } \lambda_{E} \in \mathbb{C}\right\} .
$$

Clearly any weakly closed associative ideal $\mathcal{I}$ is a weakly closed Lie ideal. It will follow from Theorem 12 that $\mathfrak{D}_{\mathcal{I}}$ corresponds to the maximal diagonal subalgebra of $\mathfrak{D}_{\mathcal{N}}$ that we can "add" to $\mathcal{I}$ to produce a Lie ideal $\mathfrak{L}$ whose canonically associated ideal $\mathcal{K}$ is the original ideal $\mathcal{I}$.

Proposition 9. If $\mathcal{I}$ is a weakly closed ideal of $\mathcal{T}(\mathcal{N})$, then $\mathfrak{D}_{\mathcal{I}}$ is a von Neumann algebra.

Proof. Given $E<F$ in $\mathcal{N}$, set $P=F-E$ and define

$$
A_{P}=\left\{T \in \mathcal{B}(\mathfrak{H}) \mid P T P=\lambda_{T} P \text { for some } \lambda_{T} \in \mathbb{C}\right\} .
$$

Clearly each $A_{P}$ is a von Neumann algebra, and so

$$
\Phi=\bigcap\left\{A_{P} \mid P=E-\widetilde{E} \text { for } E \in \mathcal{N} \text { with } \widetilde{E}<E_{-}\right\}
$$

is also a von Neumann algebra. Since $\mathfrak{D}_{\mathcal{I}}=\mathfrak{D}(\mathcal{N}) \cap \Phi$, it follows that $\mathfrak{D}_{\mathcal{I}}$ is a von Neumann algebra.

Lemma 10. Let $\mathcal{I}$ be a weakly closed associative ideal of the nest algebra $\mathcal{T}(\mathcal{N})$ and let $\mathfrak{D}_{\mathcal{I}}$ be as above. Define

$$
\begin{aligned}
\mathfrak{M}:=\left\{T \in \mathcal{T}(\mathcal{N}) \mid \text { for all } E \in \mathcal{N} \text { for which } \widetilde{E}<E_{-}\right. \text {we have } \\
\left.\qquad(E-\widetilde{E}) T(E-\widetilde{E})=\lambda_{E}(E-\widetilde{E}) \text { for some } \lambda_{E} \in \mathbb{C}\right\} .
\end{aligned}
$$

Then $\mathcal{I}+\mathfrak{D}_{\mathcal{I}}=\mathfrak{M}$. Consequently, $\mathcal{I}+\mathfrak{D}_{\mathcal{I}}$ is weakly closed.

Proof. Write $T \in \mathcal{I}+\mathfrak{D}_{\mathcal{I}}$ as $K+D$, where $K \in \mathcal{I}$ and $D \in \mathfrak{D}_{\mathcal{I}}$. Suppose that $\widetilde{E}<E_{-}$. Then $(E-\widetilde{E}) K(E-\widetilde{E})=(I-\widetilde{E}) K E=0$ and $(E-\widetilde{E}) D(E-\widetilde{E})=$ $\lambda_{E}(E-\widetilde{E})$. Thus, $T=D+K \in \mathfrak{M}$, and so $\mathcal{I}+\mathfrak{D}_{\mathcal{I}} \subseteq \mathfrak{M}$.

Conversely, suppose that $T \in \mathfrak{M}$. Let $\pi$ be any expectation of $\mathcal{B}(\mathfrak{H})$ onto $\mathfrak{D}(\mathcal{N})$. Let $D=\pi(T)$ and $K=T-\pi(T)$; clearly $T=D+K$.

We claim that $K \in \mathcal{I}$ and $D \in \mathfrak{D}_{\mathcal{I}}$. To see this, let $E \in \mathcal{N}$, and so

$$
\begin{aligned}
(E-\widetilde{E}) K(E-\widetilde{E}) & =(E-\widetilde{E})(T-\pi(T))(E-\widetilde{E}) \\
& =(E-\widetilde{E}) T(E-\widetilde{E})-(E-\widetilde{E}) \pi(T)(E-\widetilde{E}) \\
& =(E-\widetilde{E}) T(E-\widetilde{E})-\pi((E-\widetilde{E}) T(E-\widetilde{E}))
\end{aligned}
$$

If $\widetilde{E}=E_{-}$, then $\left(E-E_{-}\right) T\left(E-E_{-}\right)$belongs to the atomic part of $\mathfrak{D}(\mathcal{N})$, and so, by (1),

$$
(E-\widetilde{E}) K(E-\widetilde{E})=\left(E-E_{-}\right) T\left(E-E_{-}\right)-\left(E-E_{-}\right) T\left(E-E_{-}\right)=0 .
$$

If $\widetilde{E}<E_{-}$, then, since $T \in \mathfrak{M}$ and by (1),

$$
(E-\widetilde{E}) K(E-\widetilde{E})=\lambda_{E}(E-\widetilde{E})-\pi\left(\lambda_{E}(E-\widetilde{E})\right)=0 .
$$

Finally, if $E=\widetilde{E}$, then since $K \in \mathcal{T}(\mathcal{N}),(I-\widetilde{E}) K E=(I-E) K E=0$. Thus $(E-\widetilde{E}) K(E-\widetilde{E})=0$ for all $E$, and so $K \in \mathcal{I}$. 
As in the proof of Proposition 9, $\mathfrak{M}$ is clearly a subspace of $\mathcal{T}(\mathcal{N})$. Since $T, K \in$ $\mathfrak{M}$ and $D \in \mathfrak{D}_{\mathcal{I}}$, we have that $D=T-K \in \mathfrak{M} \cap \mathfrak{D}(\mathcal{N})=\mathfrak{D}_{\mathcal{I}}$. Thus, $T=K+D \in$ $\mathcal{I}+\mathfrak{D}_{\mathcal{I}}$ and so $\mathfrak{M} \subseteq \mathcal{I}+\mathfrak{D}_{\mathcal{I}}$. Since $\mathfrak{M}$ is easily seen to be weakly closed, it follows that $\mathcal{I}+\mathfrak{D}_{\mathcal{I}}$ is as well.

Proposition 11. If $\mathcal{I}$ is a weakly closed ideal of $\mathcal{T}(\mathcal{N})$, then $\mathcal{I}+\mathfrak{D}_{\mathcal{I}}$ is a weakly closed Lie ideal of $\mathcal{T}(\mathcal{N})$.

Proof. By Lemma $10, \mathcal{I}+\mathfrak{D}_{\mathcal{I}}$ is weakly closed. To prove that it is a Lie ideal, let $T \in \mathcal{T}(\mathcal{N})$ and $J \in \mathcal{I}+\mathfrak{D}_{\mathcal{I}}$. Then, if $E \in \mathcal{N}$ and $\widetilde{E}<E_{-}$,

$$
\begin{aligned}
(E-\widetilde{E})[T, J](E-\widetilde{E}) & =[(E-\widetilde{E}) T(E-\widetilde{E}),(E-\widetilde{E}) J(E-\widetilde{E})] \\
& =\left[(E-\widetilde{E}) T(E-\widetilde{E}), \lambda_{E}(E-\widetilde{E})\right] \\
& =0 .
\end{aligned}
$$

By Lemma $10,[T, J] \in \mathcal{I}+\mathfrak{D}_{\mathcal{I}}$, and so $\mathcal{I}+\mathfrak{D}_{\mathcal{I}}$ is a weakly closed Lie ideal of $\mathcal{T}(\mathcal{N})$.

We are now in a position to prove our main result.

Theorem 12. Let $\mathfrak{L}$ be a weakly closed Lie ideal of $\mathcal{T}(\mathcal{N})$ and $\mathcal{K}$ the associative ideal corresponding to $\mathfrak{L}$. Then $\mathcal{K} \subseteq \mathfrak{L} \subseteq \mathcal{K}+\mathfrak{D}_{\mathcal{K}}$.

Proof. Proposition 5 proves the first containment. For the second, let $L \in \mathfrak{L}$. By Proposition 8, $\mathfrak{L} \subseteq \mathcal{K}+\mathfrak{D}(\mathcal{N})$, so write $L=K+D$ for some $K \in \mathcal{K}$ and $D \in \mathfrak{D}(\mathcal{N})$. Let $E \in \mathcal{N}$ and suppose that $\widetilde{E}<E_{-}$. Consider any $F \in \mathcal{N}$ with $\widetilde{E}<F<E$ and any $T \in \mathcal{T}(\mathcal{N})$ with $T=(F-\widetilde{E}) T(E-F)$. Then since $\mathfrak{L}$ is a Lie ideal, we have $[T, L] \in \mathfrak{L}$, i.e., $[T, K]+[T, D] \in \mathfrak{L}$. Since $K \in \mathcal{K} \subseteq \mathfrak{L}$, we have $[T, K] \in \mathfrak{L}$, and so $[T, D] \in \mathfrak{L} \subseteq \mathcal{K}+\mathfrak{D}(\mathcal{N})$.

Now if $\bar{K}_{1}+D_{1} \in \mathcal{K}+\mathfrak{D}(\mathcal{N})$ and $E, F$ are as above, then since $D_{1} \in \mathcal{N}^{\prime}$, we have

$$
\begin{aligned}
(F-\widetilde{E})\left(K_{1}+D_{1}\right)(E-F) & =(F-\widetilde{E}) K_{1}(E-F)+(F-\widetilde{E}) D_{1}(E-F) \\
& =(F-\widetilde{E})(I-\widetilde{E}) K_{1} E(E-F) \\
& =0 .
\end{aligned}
$$

Hence, since $[T, D] \in \mathcal{K}+\mathfrak{D}(\mathcal{N})$, it follows that $(F-\widetilde{E})[T, D](E-F)=0$. Thus

$$
\begin{aligned}
0 & =(F-\widetilde{E})[T, D](E-F) \\
& =(F-\widetilde{E}) T D(E-F)-(F-\widetilde{E}) D T(E-F) \\
& =T D(E-F)-(F-\widetilde{E}) D T \\
& =T((E-F) D(E-F))-((F-\widetilde{E}) D(F-\widetilde{E})) T
\end{aligned}
$$

and so

$$
T((E-F) D(E-F))=((F-\widetilde{E}) D(F-\widetilde{E})) T
$$

for all $T \in \mathcal{B}(\mathfrak{H})$ for which $T=(F-\widetilde{E}) T(E-F)$. Hence, we conclude that

$$
D(F-\widetilde{E})=\lambda_{E}(F-\widetilde{E}) \quad \text { and } \quad D(E-F)=\lambda_{E}(E-F)
$$

for some $\lambda_{E} \in \mathbb{C}$. But this is true for all $F$ satisifying $\widetilde{E}<F<E$, and so this implies that $D(E-\widetilde{E})=\lambda_{E}(E-\widetilde{E})$. Since this in turn is true for all $E \in \mathcal{N}$ with $\widetilde{E}<E_{-}$, we conclude that $D \in \mathfrak{D}_{\mathcal{K}}$. Thus, $L \in \mathcal{K}+\mathfrak{D}_{\mathcal{K}}$, i.e., $\mathfrak{L} \subseteq \mathcal{K}+\mathfrak{D}_{\mathcal{K}}$. 
Remark 1. Let $\mathcal{K}$ be a weakly closed associative ideal of $\mathcal{T}(\mathcal{N})$. It is not true that every weakly closed subspace $S$ of $\mathcal{T}(\mathcal{N})$ satisfying $\mathcal{K} \subseteq S \subseteq \mathcal{K}+\mathfrak{D}_{\mathcal{K}}$ is a Lie ideal. Indeed, consider the trivial nest $\{0, \mathfrak{H}\}$ for any Hilbert space of dimension larger than 1 and $\mathcal{K}=\{0\}$. In this case $\mathfrak{D}_{\mathcal{K}}=\mathcal{B}(\mathfrak{H})$, and the span of any nontrivial projection is a counterexample to the above statement.

Remark 2. The compression of a Lie ideal $\mathfrak{L}$ of $\mathcal{T}(\mathcal{N})$ to any interval (in particular to an atom) must also be a Lie ideal. Since it must also be weakly closed, the compression of $\mathfrak{L}$ to an atom must be one of $\{0\}, \mathbb{C} I$, or $\mathrm{sl}_{n}$ (the elements of trace zero) if the atom is finite dimensional, or $\mathcal{B}(\mathfrak{H})$ for the (finite or infinite dimensional) atom $\mathfrak{H}$ (see [3]). The following example shows that the converse is false. That is, if $\mathcal{K}$ is a weakly closed associative ideal and $S$ a weakly closed subspace with $\mathcal{K} \subseteq S \subseteq \mathcal{K}+\mathfrak{D}_{\mathcal{K}}$ and so that the compression of $\mathrm{S}$ to every atom in $\mathcal{N}$ is a Lie ideal, it does not follow that $S$ itself is Lie ideal.

Example 13. There exists a weakly closed subspace $S$ of $\mathcal{T}(\mathcal{N})$ satisfying:

(i) $\mathcal{I} \subseteq S \subseteq \mathcal{I}+\mathfrak{D}_{\mathcal{I}}$ for some weakly closed associative ideal $\mathcal{I}$;

(ii) the restriction of $S$ to every atom of $\mathcal{N}$ is a Lie ideal,

but $S$ itself is not a Lie ideal.

Let $\mathfrak{H}=\mathbb{C}^{4}, \mathcal{N}=\left\{\{0\}, \operatorname{span}\left\{e_{1}, e_{2}\right\}, \mathfrak{H}\right\}$, and $\mathcal{I}=P \mathcal{T}(\mathcal{N})(I-P)$, where $P$ is the orthogonal projection onto $\operatorname{span}\left\{e_{1}, e_{2}\right\}$. Thus,

$$
\mathcal{T}(\mathcal{N})=\left[\begin{array}{cccc}
* & * & * & * \\
* & * & * & * \\
0 & 0 & * & * \\
0 & 0 & * & *
\end{array}\right] \quad \text { and } \quad \mathcal{I}=\left[\begin{array}{cccc}
0 & 0 & * & * \\
0 & 0 & * & * \\
0 & 0 & 0 & 0 \\
0 & 0 & 0 & 0
\end{array}\right]
$$

and moreover, $\mathfrak{D}_{\mathcal{I}}=\mathbf{M}_{2} \oplus \mathbf{M}_{2}$ and $\mathcal{I}+\mathfrak{D}_{\mathcal{I}}=\mathcal{T}(\mathcal{N})$. Let

$$
\mathcal{S}=\left\{\left[\begin{array}{cc}
X & A \\
0 & X
\end{array}\right] \mid X, A \in \mathbf{M}_{2}\right\},
$$

so that $\mathcal{I} \subseteq \mathcal{S} \subseteq \mathcal{I}+\mathfrak{D}_{\mathcal{I}}$ and $\mathcal{S}$ is weakly closed. Then

$$
S=\left[\begin{array}{cccc}
0 & 0 & 0 & 0 \\
1 & 0 & 0 & 0 \\
0 & 0 & 0 & 0 \\
0 & 0 & 1 & 0
\end{array}\right] \in \mathcal{S} \quad \text { and } \quad T=\left[\begin{array}{cccc}
3 & 0 & 0 & 0 \\
0 & 3 & 0 & 0 \\
0 & 0 & 0 & 1 \\
0 & 0 & 0 & 0
\end{array}\right] \in \mathcal{T}(\mathcal{N})
$$

but since

$$
[S, T]=\left[\begin{array}{cccc}
0 & 0 & 0 & 0 \\
0 & 0 & 0 & 0 \\
0 & 0 & -1 & 0 \\
0 & 0 & 0 & 1
\end{array}\right] \notin \mathcal{S}
$$

we find that $\mathcal{S}$ is not a Lie ideal.

For every associative ideal $\mathcal{I}$, there exists a Lie ideal $\mathcal{I}_{Z}$ such that every linear manifold between $\mathcal{I}$ and $\mathcal{I}_{Z}$ is a Lie ideal.

Definition. Let $\mathcal{I}$ be an associative ideal of $\mathcal{T}(\mathcal{N})$. Define

$$
\mathcal{I}_{Z}=\{X \in \mathcal{T}(\mathcal{N}) \mid[A, X] \in \mathcal{I} \quad \forall A \in \mathcal{T}(\mathcal{N})\} .
$$


Note that $\mathcal{I}_{Z}$ is simply the lifting of the centre of $\mathcal{T}(\mathcal{N}) / \mathcal{I}$, and that it clearly has the above mentioned property. Example 13 shows that, in general, $\mathcal{I}+\mathfrak{D}_{\mathcal{I}}$ need not be contained in $\mathcal{I}_{Z}$.

Recall that a nest $\mathcal{N}$ is called maximal if all of its atoms have dimension one. In particular, it follows that a continuous nest is maximal. In contrast to the previous example, we have:

Proposition 14. Let $\mathcal{N}$ be a nest and $\mathcal{I}$ a weakly closed associative ideal of $\mathcal{T}(\mathcal{N})$. Then

(i) $\mathcal{I}_{Z} \subseteq \mathcal{I}+\mathfrak{D}_{\mathcal{I}}$, and

(ii) if $\mathcal{N}$ is maximal, then $\mathcal{I}_{Z}=\mathcal{I}+\mathfrak{D}_{\mathcal{I}}$; in particular, every linear manifold between $\mathcal{I}$ and $\mathcal{I}+\mathfrak{D}_{\mathcal{I}}$ is a Lie ideal.

Proof. For (i), let $R \in \mathcal{I}_{Z}$ and $E \in \mathcal{N}$ with $\widetilde{E}<E_{-}$. Since $[R, T] \in \mathcal{I}$ for every $T \in \mathcal{T}(\mathcal{N})$, then by Lemma 3

$$
0=(E-\widetilde{E})[R, T](E-\widetilde{E})=[(E-\widetilde{E}) R(E-\widetilde{E}),(E-\widetilde{E}) T(E-\widetilde{E})] .
$$

Thus, $(E-\widetilde{E}) R(E-\widetilde{E})$ lies in the commutant of the compression of $\mathcal{T}(\mathcal{N})$ to $(E-\widetilde{E}) \mathfrak{H}$, which is itself a nest algebra. Since the commutant of a nest algebra is well-known to be trivial [1, Corollary 19.5], we find that $(E-\widetilde{E}) R(E-\widetilde{E})=$ $\lambda_{E}(E-\widetilde{E})$ for some $\lambda_{E} \in \mathbb{C}$. Thus $R \in \mathcal{I}+\mathfrak{D}_{\mathcal{I}}$.

To prove (ii), suppose $\mathcal{N}$ is maximal and let $L \in \mathcal{I}+\mathfrak{D}_{\mathcal{I}}$. Then $L=K+D$ for some $K \in \mathcal{I}$ and $D \in \mathfrak{D}_{\mathcal{I}}$. Let $T \in \mathcal{T}(\mathcal{N})$, so $[T, L]=[T, K]+[T, D]$. Since $K \in \mathcal{I}$ and $\mathcal{I}$ is an ideal, we have $[T, K] \in \mathcal{I}$. Thus it suffices to show that $[T, D] \in \mathcal{I}$.

First observe that for all $E \in \mathcal{N}$, we have $(E-\widetilde{E}) D(E-\widetilde{E})=\lambda_{E}(E-\widetilde{E})$ for some $\lambda_{E} \in \mathbb{C}$. Indeed, this follows from the definition of $\mathfrak{D}_{\mathcal{I}}$ if $\widetilde{E}<E_{-}$, and if $\widetilde{E}=E_{-}$, then $E-\widetilde{E}$ is one dimensional by virtue of the maximality of the nest. Therefore,

$$
\begin{aligned}
(E-\widetilde{E})[T, D](E-\widetilde{E}) & =[(E-\widetilde{E}) T(E-\widetilde{E}),(E-\widetilde{E}) D(E-\widetilde{E})] \\
& =\left[(E-\widetilde{E}) T(E-\widetilde{E}), \lambda_{E}(E-\widetilde{E})\right]=0 .
\end{aligned}
$$

Thus by Lemma $3,[T, D] \in \mathcal{I}$ and so $[T, L] \in \mathcal{I}$. This proves that $L \in \mathcal{I}_{Z}$, i.e., $\mathcal{I}+\mathfrak{D}_{\mathcal{I}} \subseteq \mathcal{I}_{Z}$.

In the finite dimensional case of Theorem 1, notice that the associative ideal $\mathcal{K}$ corresponding to a Lie ideal $\mathfrak{L}$ of $\mathbf{T}_{n}$ is diagonal disjoint. That is, if $\pi$ is the projection onto the diagonal, then $\pi(K)=0$ for all $K \in \mathcal{K}$. A similar decomposition of a Lie ideal into the direct sum of its diagonal and off-diagonal parts holds for triangular UHF algebras (see Section 2). The situation for nest algebras depends on the nature of the nest, as the following results demonstrate.

Recall that if $x, y \in \mathfrak{H}$, then the rank-one operator $x \otimes y^{*}$ is defined by $x \otimes y^{*}(z)=$ $\langle z, y\rangle x, z \in \mathfrak{H}$.

Proposition 15. Let $\mathcal{N}$ be the Volterra nest and let $\pi$ be any expectation of $\mathcal{T}(\mathcal{N})$ onto the diagonal $\mathfrak{D}(\mathcal{N})$. Then

$$
\overline{\{x-\pi(x) \mid x \in \mathcal{T}(\mathcal{N})\}}^{w}=\mathcal{T}(\mathcal{N}) .
$$

Proof. Let $\mathcal{U}=\overline{\{x-\pi(x) \mid x \in \mathcal{T}(\mathcal{N})\}}^{w}$. If $x \otimes y^{*} \in \mathcal{T}(\mathcal{N})$, then there is some $N \in \mathcal{N}$ so that $x \in N$ and $y \in N^{\perp}$. Thus $\pi\left(x \otimes y^{*}\right)=0$, and so $x \otimes y^{*}-\pi\left(x \otimes y^{*}\right)=$ 
$x \otimes y^{*}$. This shows that $\mathcal{U}$ contains all rank-one operators in $\mathcal{T}(\mathcal{N})$. It follows that $\mathcal{U}$ contains all finite ranks in $\mathcal{T}(\mathcal{N})$, and since $\mathcal{U}$ is weakly closed, we have $\mathcal{U}=\mathcal{T}(\mathcal{N})$.

From this we see that if $\mathfrak{L}=\mathcal{T}(\mathcal{N})$, where $\mathcal{N}$ is the Volterra nest, then the corresponding associative ideal $\mathcal{K}$ is $\mathcal{T}(\mathcal{N})=\mathfrak{L}$. Since $\mathfrak{D}_{\mathcal{K}}$ is then equal to $\mathfrak{D}(\mathcal{N})$, no direct sum decomposition of $\mathfrak{L}$ is possible (compare with Proposition 18).

Example 16. Consider a nest $\mathcal{N}$. The atomic part of the diagonal of $\mathcal{N}$ is the von Neumann subalgebra $\mathfrak{D}_{a}=\sum \oplus \mathcal{B}\left(H_{a}\right)$, where the direct sum is taken over the set of atoms $H_{a}$ of the nest. Let $\pi_{a}$ denote the homomorphism from $\mathcal{T}(\mathcal{N})$ onto the atomic part of the diagonal. If $\mathfrak{L}$ is a Lie ideal, it is in general false that $\pi_{a}(L) \in \mathfrak{L}$ for all $L \in \mathfrak{L}$. Indeed, recall that given a nest $\mathcal{N}$, there is an order preserving homeomorphism of $\mathcal{N}$ onto a compact subset of $[0,1]$, which allows one to talk about an order type of a nest [1, Theorem 2.13]. As such we may consider a nest $\mathcal{N}$ of order type $\left[0, \frac{1}{3}\right] \cup\left\{\frac{1}{2}\right\} \cup\left[\frac{2}{3}, 1\right]$. Let $\mathfrak{L}=\mathbb{C} I$, and notice that $\mathcal{K}=\{0\}$. Then $I \in \mathfrak{L}$, yet $\pi_{a}(I)=0 \oplus 1 \oplus 0 \notin \mathfrak{L}$.

One may also ask to what extent the decomposition in Theorem 12 is unique. We have the following result.

Proposition 17. Suppose $\mathfrak{L}$ is a weakly closed Lie ideal of $\mathcal{T}(\mathcal{N})$ and $\mathcal{K}_{1}, \mathcal{K}_{2}$ are two weakly closed associative ideals of $\mathcal{T}(\mathcal{N})$ such that

$$
\mathcal{K}_{1} \subseteq \mathfrak{L} \subseteq \mathcal{K}_{1}+\mathfrak{D}(\mathcal{N}) \quad \text { and } \quad \mathcal{K}_{2} \subseteq \mathfrak{L} \subseteq \mathcal{K}_{2}+\mathfrak{D}(\mathcal{N})
$$

If $\pi$ is any expectation onto $\mathfrak{D}(\mathcal{N})$, then $\mathcal{K}_{1} \cap \operatorname{ker} \pi=\mathcal{K}_{2} \cap \operatorname{ker} \pi$.

Proof. Using Theorem 2, let $\mathcal{K}_{1}$ be defined by the function $E \mapsto \widetilde{E}$ and $\mathcal{K}_{2}$ be defined by the function $E \mapsto \widehat{E}$. Assume that $\mathcal{K}_{1} \cap \operatorname{ker} \pi \neq \mathcal{K}_{2} \cap \operatorname{ker} \pi$. Without loss of generality, there exists some $T \in \mathcal{K}_{2} \backslash \mathcal{K}_{1}$ with $T \in \operatorname{ker} \pi$. Thus there is $E \in \mathcal{N}$ such that $(I-\widehat{E}) T E=0$ but $(I-\widetilde{E}) T E \neq 0$. Thus $\widetilde{E}<\widehat{E}$, and since $\mathcal{K}_{2}$ is an ideal, $0 \neq(\widehat{E}-\widetilde{E}) T E \in \mathcal{K}_{2}$.

If $\widehat{E}<E$, then $(\widehat{E}-\widetilde{E}) T E$ has zero $\pi$-diagonal, but $(\widehat{E}-\widetilde{E}) T E \notin \mathcal{K}_{1}$, and so $(\widehat{E}-\widetilde{E}) T E \notin \mathcal{K}_{1}+\mathfrak{D}(\mathcal{N})$, a contradiction since $(\widehat{E}-\widetilde{E}) T E \in \mathcal{K}_{2} \subseteq \mathfrak{L} \subseteq \mathcal{K}_{1}+\mathfrak{D}(\mathcal{N})$.

If $\widehat{E}=E$, then either (i) $\widetilde{E}<E_{-}$or (ii) $\widetilde{E}=E_{-}$. If (i) holds, then there is some $N \in \mathcal{N}$ with $\widetilde{E}<N<E$ and so that $(N-\widetilde{E}) T(E-N) \neq 0$. Again, this has zero diagonal, but it does not belong to $\mathcal{K}_{1}$. Thus, $(N-\widetilde{E}) T(E-N) \notin \mathcal{K}_{1}+\mathfrak{D}(\mathcal{N})$, a contradiction as above.

Finally, if (ii) holds, then $0 \neq(\widehat{E}-\widetilde{E}) T E=\left(E-E_{-}\right) T\left(E-E_{-}\right) \in \mathfrak{D}(\mathcal{N})$. But

$$
\begin{aligned}
\left(E-E_{-}\right) T\left(E-E_{-}\right) & =\pi\left(\left(E-E_{-}\right) T\left(E-E_{-}\right)\right) \\
& =\left(E-E_{-}\right) \pi(T)\left(E-E_{-}\right) \\
& =0,
\end{aligned}
$$

a contradiction. Thus, $\mathcal{K}_{1} \cap \operatorname{ker} \pi=\mathcal{K}_{2} \cap \operatorname{ker} \pi$.

If $\mathcal{N}$ is an atomic nest, there is a unique expectation of $\mathcal{T}(\mathcal{N})$ onto $\mathfrak{D}(\mathcal{N})[1, \mathrm{p}$. 90]. The final result of this section shows that when the underlying nest is atomic, we can obtain a decomposition of a weakly closed Lie ideal of the nest algebra as the direct sum of its diagonal and off-diagonal parts, paralleling the situation in the finite dimensional case. 
Proposition 18. Let $\mathcal{N}$ be an atomic nest, $\mathfrak{L}$ a weakly closed Lie ideal of $\mathcal{T}(\mathcal{N})$, and $\mathcal{K}$ the weakly closed associative ideal of $\mathcal{T}(\mathcal{N})$ corresponding to $\mathfrak{L}$. If $\pi$ denotes the unique expectation of $\mathcal{T}(\mathcal{N})$ onto the diagonal $\mathfrak{D}(\mathcal{N})$, then

$$
\mathcal{K}=\overline{\{x-\pi(x) \mid x \in \mathfrak{L}\}}^{w} .
$$

In particular, $\mathfrak{L}=\mathcal{K} \oplus \pi(\mathfrak{L})$.

Proof. Set $\mathfrak{J}=\overline{\{x-\pi(x) \mid x \in \mathfrak{L}\}}^{\mathrm{w}}$. To prove the proposition, it suffices to show that $\mathfrak{J}$ is a weakly closed associative ideal of $\mathcal{T}(\mathcal{N})$ such that $\mathfrak{J} \subseteq \mathfrak{L} \subseteq \mathfrak{J}+\mathfrak{D}(\mathcal{N})$. Indeed, in this case Proposition 17 gives that $\mathcal{K} \cap \operatorname{ker} \pi=\mathfrak{J} \cap \operatorname{ker} \pi$. But since $\mathcal{K} \cap \operatorname{ker} \pi=\mathcal{K}$ and $\mathfrak{J} \cap \operatorname{ker} \pi=\mathfrak{J}, \mathcal{K}=\mathfrak{J}$, completing the proof.

To show that $\mathfrak{J}$ has the desired properties, let $\mathcal{F}$ be a finite subnest of $\mathcal{N}$ and let $\left\{E_{i}\right\}_{i}$ denote the intervals of $\mathcal{F}$. If $\mathcal{G}$ is any subnest of $\mathcal{N}$ and $J \in \mathcal{T}(\mathcal{G})$, then set $L_{\mathcal{G}}(J)=\sum_{i} E_{i-1} J\left(E_{i}-E_{i-1}\right)$.

We first show that if $J \in \mathfrak{J}$ and $A \in \mathcal{T}(\mathcal{N})$, then $A L_{\mathcal{F}}(J) \in \mathfrak{L}$. Let $L=L_{\mathcal{F}}(J)$. If $i<j$, then $E_{j} L E_{i}=-\left[E_{j},\left[E_{i}, L\right]\right]$, and since $J \subseteq \mathfrak{L}$, we conclude that $E_{j} L E_{i} \in \mathfrak{L}$. Thus, for $i<j \leq k$, we have $E_{k} A E_{j} L E_{i}=\left[E_{k} A E_{j}, E_{j} L E_{i}\right] \in \mathfrak{L}$. If $i \geq j$, then $E_{j} L E_{i}=0$, and if $k>j$, then $E_{k} A E_{j}=0$. As such, $E_{k} A E_{j} L E_{i} \in \mathfrak{L}$ for all $i, j$, and $k$. Summing over all such $i, j, k$, we obtain that $A L=A L_{\mathcal{F}}(J) \in \mathfrak{L}$.

If $X \in \mathfrak{L}$, then $2 X-\sum_{i}\left[E_{i},\left[E_{i}, X\right]\right] \in \mathfrak{L}$. But

$$
\begin{aligned}
2 X-\sum_{i}\left[E_{i},\left[E_{i}, X\right]\right] & =2 X-\sum_{i} E_{i} X-E_{i} X E_{i}-E_{i} X E_{i}+X E_{i} \\
& =2 \sum_{i} E_{i} X E_{i} \\
& =2 \pi_{\mathcal{F}}(X),
\end{aligned}
$$

where $\pi_{\mathcal{F}}$ is the projection onto $\mathfrak{D}(\mathcal{F})$. It follows that $\pi_{\mathcal{F}}(X)$ belongs to $\mathfrak{L}$. As $\mathfrak{L}$ is weakly closed, it is also SOT-closed. Since $\mathcal{N}$ is atomic, it follows from $[1$, Proposition 4.4] that $\pi(X)=$ sот- $\lim _{\mathcal{F}} \pi_{\mathcal{F}}(X) \in \mathfrak{L}$. Thus $\mathfrak{J} \subseteq \mathfrak{L}$.

We claim that $J=$ оот- $\lim _{\mathcal{F}} L$. Now $\pi(J)=\operatorname{sot}_{-} \lim _{\mathcal{F}} \pi_{\mathcal{F}}(J)$ [1, Proposition 4.4], and thus, by [1, Proposition 4.5], $L_{\mathcal{N}}(J)=\operatorname{sor}^{-} \lim _{\mathcal{F}} L=J-\pi(J)$. Let $x, y \in \mathfrak{H}_{a}$ for any atom $\mathfrak{H}_{a}$ of $\mathcal{N}$. By definition, we can find a weakly convergent net $\left\{J_{\alpha}\right\} \subseteq \mathfrak{L}$ such that

$$
\langle\pi(J) x, y\rangle=\lim _{\alpha}\left\langle\left(J_{\alpha}-\pi\left(J_{\alpha}\right)\right) x, y\right\rangle=0 .
$$

Thus $\pi(J)=0$, and so $J=J-\pi(J)$, proving our claim.

It is now immediate from the above that if $A \in \mathcal{T}(\mathcal{N})$, then $A J=$ sот- $^{-\lim _{\mathcal{F}}} A L$. But for all $\mathcal{F}^{\prime} \geq \mathcal{F}, L_{\mathcal{F}^{\prime}}\left(A L_{\mathcal{F}}(J)\right)=A L_{\mathcal{F}}(J)$, so

$$
A J=\text { sот- } \lim _{\mathcal{F}} A L=\text { sот- } \lim _{\mathcal{F}^{\prime}}\left(\text { sот- } \lim _{\mathcal{F}} L_{\mathcal{F}^{\prime}}\left(A L_{\mathcal{F}}(J)\right)\right) \in \mathfrak{J},
$$

and so $\mathfrak{J}$ is an ideal.

\section{Norm-Closed Lie ideals in triangular UHF algebras}

Let $\left\{p_{n}\right\}$ be an increasing sequence of positive integers such that $p_{n} \mid p_{n+1}$ for each $n \geq 1$. Consider a sequence of $C^{*}$-algebras $\mathbb{A}_{n} \simeq^{*} \mathbb{M}_{p_{n}}$ and $*$-homorphisms $\phi_{n}: \mathbb{A}_{n} \rightarrow \mathbb{A}_{n+1}$. The $C^{*}$-algebra inductive limit $\mathbb{A}$ of the system $\left\{\left(\mathbb{A}_{n}, \phi_{n}\right)\right\}$ is called a uniformly hyperfinite or UHF algebra. Alternatively, $\mathbb{A}$ is a UHF algebra if there exists an increasing sequence $\left\{\mathbb{A}_{n}\right\}_{n}$ of full matrix algebras whose union is dense in $\mathbb{A}$. 
Let $\mathfrak{D}$ be a maximal abelian self-adjoint subalgebra (i.e. a masa) of a UHF algebra $\mathbb{A}$, and let $\mathcal{C}$ be any subset of $\mathbb{A}$. The normalizer of $\mathfrak{D}$ in $\mathcal{C}$ is the set

$$
\mathcal{N}_{\mathfrak{D}}(\mathcal{C})=\left\{w \in \mathcal{C} \mid w \text { is a partial isometry, } w \mathfrak{D} w^{*} \subseteq \mathfrak{D}, w^{*} \mathfrak{D} w \subseteq \mathfrak{D}\right\} .
$$

$\mathfrak{D}$ is said to be a canonical masa if there exists an increasing sequence $\left\{\mathbb{A}_{n}\right\}_{n}$ of full matrix algebras whose union is dense in $\mathbb{A}$ such that $\mathfrak{D}_{n}=\mathfrak{D} \cap \mathbb{A}_{n}$ is a masa in $\mathbb{A}_{n}, \mathfrak{D}=\overline{\bigcup_{n} \mathfrak{D}_{n}}$, and $\mathcal{N}_{\mathfrak{D}_{n}}\left(\mathbb{A}_{n}\right) \subseteq \mathcal{N}_{\mathfrak{D}_{n+1}}\left(\mathbb{A}_{n+1}\right)$ for all $n \geq 1$.

Definition. A triangular UHF (TUHF) algebra $\mathcal{Q}$ is the Banach algebra direct limit of a system

$$
\mathcal{Q}_{1} \stackrel{\varphi_{1}}{\longrightarrow} \mathcal{Q}_{2} \stackrel{\varphi_{2}}{\longrightarrow} \mathcal{Q}_{3} \stackrel{\varphi_{3}}{\longrightarrow} \mathcal{Q}_{4} \cdots,
$$

where $\mathcal{Q}_{n}$ is isometrically isomorphic to some full upper triangular matrix algebra $\mathbf{T}_{p_{n}}$ and $\varphi_{n}: \mathcal{Q}_{n} \rightarrow \mathcal{Q}_{n+1}$ is an embedding, i.e. the restriction of a $C^{*}$-isomorphism, so that the extension of $\varphi_{n}$ carries $\mathcal{N}_{\mathfrak{D}_{n}}\left(\mathbb{A}_{n}\right)$ into $\mathcal{N}_{\mathfrak{D}_{n+1}}\left(\mathbb{A}_{n+1}\right)$.

These algebras are strongly maximal, in the sense that $\mathcal{Q}+\mathcal{Q}^{*}=\mathbb{A}$. They are also called strongly maximal triangular in factors [13].

We denote the direct limit of the system $(2)$ by $\lim \left(\mathcal{Q}_{n} ; \varphi_{n}\right)$, and call it a presentation for the algebra. For $k \leq n$, let $\varphi_{n, k}: \overrightarrow{\mathcal{Q}_{k}} \rightarrow \mathcal{Q}_{n}$ be the composition $\varphi_{n-1} \circ \cdots \circ \varphi_{k}$.

Our present goal is to investigate the Lie ideal structure of TUHF algebras. We show that to each norm-closed Lie ideal $\mathfrak{L}$ of a triangular UHF algebra $\mathcal{Q}$ there correspond an associative ideal $\mathcal{K}$ and a diagonal $C^{*}$-algebra $\mathfrak{D}_{\mathcal{K}}$ such that $\mathcal{K} \subseteq \mathfrak{L} \subseteq \mathfrak{D}_{\mathcal{K}}+\mathcal{K}$.

We emphasize that, in general, the nest algebra definition of the ideal $\mathcal{K}$ is inappropriate in the limit algebra case. Indeed, let $\sigma_{n}: \mathbf{T}_{2^{n}} \rightarrow \mathbf{T}_{2^{n+1}}$ denote the standard embedding, i.e., the map defined by $\sigma_{n}(a)=a \oplus a, a \in \mathbf{T}_{2^{n}}$. Set $\mathcal{A}=$ $\lim \left(\mathbf{T}_{2_{n}}, \sigma_{n}\right)$, the $2^{\infty}$ standard TUHF algebra. It is well-known that Lat $\mathcal{A}$ is trivial, so the ideal $\mathcal{K}$ obtained via the definition before Lemma 4 is the zero ideal while clearly any nonzero associative ideal gives a Lie ideal that is nonzero off the diagonal. Actually, Propositions 18, 21 and 22 suggest that the limit algebra case closely parallels the atomic nest setting, as one might expect.

Throughout the following, let $\mathcal{Q}$ be a triangular UHF algebra with presentation $\lim \left(\mathcal{Q}_{n}, \varphi_{n}\right)$, where each $\mathcal{Q}_{n}$ is isometrically isomorphic to $\mathbf{T}_{p_{n}}$ for some positive integer $p_{n}$. Let $\mathbb{A}$ denote the corresponding UHF algebra and $\mathfrak{D}$ the canonical masa $\mathcal{Q} \cap \mathcal{Q}^{*}$. We denote by $\pi$ the contractive projection of $\mathbb{A}$ onto $\mathfrak{D}[14$, Proposition 4.1].

We begin with two elementary lemmas. The first is actually Lemma 2.2 of [9], but we reproduce it here in order to keep the paper as self-contained as possible.

Lemma 19. Let $\mathbb{B}$ be a unital complex algebra and suppose that $\mathfrak{L}$ is a Lie ideal of $\mathbf{M}_{k} \otimes \mathbb{B}$. If $y=\sum_{i=1}^{n} \sum_{j=1}^{n} e_{i j} \otimes y_{i j} \in \mathfrak{L}$, then $e_{i j} \otimes y_{i j} \in \mathfrak{L}$ for each $i \neq j$.

Proof. Fix $1 \leq i \leq n$. Letting $y^{\prime}=\left[y,\left(e_{i i} \otimes 1\right)\right]$ and $y^{\prime \prime}=\left[y^{\prime},\left(e_{i i} \otimes 1\right)\right]$, we have $y^{\prime}$ in $\mathfrak{L}$, and hence $y^{\prime \prime}$ belongs to $\mathfrak{L}$. As such, if $z^{\prime}=\frac{1}{2}\left(y^{\prime \prime}-y^{\prime}\right)$, then $z^{\prime} \in \mathfrak{L}$. A calculation shows that

$$
z^{\prime}=\left(\sum_{j=1}^{n} e_{i j} \otimes y_{i j}\right)-e_{i i} \otimes y_{i i} .
$$

If $j \neq i$, then $\left[z^{\prime},\left(e_{j j} \otimes 1\right)\right]=e_{i j} \otimes y_{i j} \in \mathfrak{L}$. 
Lemma 20. Let $\mathbb{B}$ be a unital complex algebra and suppose that $\mathcal{R}$ is a unital subalgebra of $\mathbf{M}_{n} \otimes \mathbb{B}$. Suppose that $\mathfrak{L}$ is a Lie ideal of $\mathcal{R}$ and that $\left\{e_{i j} \otimes 1: 1 \leq\right.$ $i \leq j \leq n\} \subseteq \mathcal{R}$. Finally, suppose $y \in \mathfrak{L}$ and $y=\sum_{i=1}^{n} \sum_{j=1}^{n} e_{i j} \otimes y_{i j}$. Then

(i) $e_{i j} \otimes y_{i j} \in \mathfrak{L}$ if $i \neq j$,

(ii) if $i<j<m \leq n$, then $e_{i m} \otimes y_{i j} \in \mathfrak{L}$, and

(iii) if $1 \leq m<i<j$, then $e_{m j} \otimes y_{i j} \in \mathfrak{L}$.

Proof. Since both $e_{i i} \otimes 1$ and $e_{j j} \otimes 1$ belong to $\mathcal{R}$, part (i) follows from the proof of Lemma 19.

By (i), $z=e_{i j} \otimes y_{i j} \in \mathfrak{L}$. Now $j<m$ implies that $e_{j m} \otimes 1 \in \mathcal{R}$, so that

$$
\left[z,\left(e_{j m} \otimes 1\right)\right]=\left[\left(e_{i j} \otimes y_{i j}\right),\left(e_{j m} \otimes 1\right)\right]=e_{i m} \otimes y_{i j} \in \mathfrak{L},
$$

proving (ii). To see (iii), observe that by (i), $z=e_{i j} \otimes y_{i j} \in \mathfrak{L}$. Since $m<i$, we have $e_{m i} \otimes 1 \in \mathcal{R}$, so that

$$
\left[\left(e_{m i} \otimes 1\right), z\right]=\left[\left(e_{m i} \otimes 1\right),\left(e_{i j} \otimes y_{i j}\right)\right]=e_{m j} \otimes y_{i j} \in \mathfrak{L} .
$$

A closed subspace $S$ of a UHF algebra $\mathbb{A}$ is said to be inductive if for every increasing chain $\left\{\mathbb{B}_{n}\right\}$ of full matrix algebras such that $\mathbb{A}=\overline{\bigcup_{n=1}^{\infty} \mathbb{B}_{n}}$, we have $S=\overline{\bigcup_{n=1}^{\infty}\left(S \cap \mathbb{B}_{n}\right)}$. In particular, every closed $\mathfrak{D}$-bimodule of $\mathbb{A}$ is inductive $[14$, Proposition 4.7].

Proposition 21. Let $\mathfrak{L}$ be a closed Lie ideal in $\mathcal{Q}$. If

$$
\mathcal{K}=\{x-\pi(x) \mid x \in \mathfrak{L}\},
$$

then $\mathcal{K}$ is a closed $\mathfrak{D}$-bimodule of $\mathcal{Q}$ contained in $\mathfrak{L}$. In particular, $\mathcal{K}$ is inductive.

Proof. Step One: First we show that $\mathcal{K}$ is a subset of $\mathfrak{L}$.

We choose a system $\left\{e_{i j}^{[k]} \mid i \leq j, k \in \mathbb{N}\right\}$ of matrix units for the triangular UHF algebra $\mathcal{Q}$; by $*$-extendibility, this naturally extends to a system of matrix units for the enveloping UHF algebra $\mathbb{A}[14]$. Recall that for each $k \geq 1$, the map

$$
\begin{aligned}
\pi_{k}: \mathbb{A} & \longrightarrow \mathfrak{D}, \\
a & \longmapsto \sum_{i} e_{i i}^{[k]} a e_{i i}^{[k]},
\end{aligned}
$$

is a contractive projection and the $\pi_{k}$ 's form a sequence converging pointwise to $\pi$, the contractive projection of $\mathbb{A}$ onto the canonical masa $\mathfrak{D}$ associated with these matrix units. Moreover, $\pi\left(d_{1} a d_{2}\right)=d_{1} \pi(a) d_{2}$ for all $d_{1}, d_{2} \in \mathfrak{D}$ and $a \in \mathbb{A}[14$, Proposition 4.1].

Let $k \geq 1$, so $p_{k}$ divides the supernatural number $\alpha$ of the UHF algebra $\mathbb{A}$. Let $\mathbb{B}_{k}$ be a UHF algebra whose supernatural number is $\beta=\alpha / p_{k}$. By Glimm's Theorem [5, Theorem 1.12] $\mathbb{A}$ is ${ }^{*}$-isomorphic to $\mathbf{M}_{p_{k}} \otimes \mathbb{B}_{k}$, and so we can think of elements of $\mathrm{A}$ as $p_{k} \times p_{k}$ matrices over the UHF algebra $\mathbb{B}_{k}$. In particular, we may do this in such a way that $e_{i j}^{[k]}$ is identified with $e_{i j}^{(k)} \otimes 1^{(k)}$, where $\left\{e_{i j}^{(k)}\right\}_{i, j=1}^{p_{k}}$ are the standard matrix units for $\mathbf{M}_{p_{k}}$, while $1^{(k)}$ denotes the identity element of $\mathbb{B}_{k}$. In general, given $m \in \mathbb{A} \simeq^{*} \mathbf{M}_{p_{k}} \otimes \mathbb{B}_{k}$, we write $m=\sum_{i, j=1}^{p_{k}} e_{i j}^{(k)} \otimes m_{i j}^{(k)}$, with each $m_{i j}^{(k)} \in \mathbb{B}_{k}$. Since $\left\{e_{i j}^{(k)}\right\}_{i, j=1}^{p_{k}}$ is a basis for $\mathbf{M}_{p_{k}}$, this representation of $m$ is unique. It will be convenient to introduce the notation $m_{i j}^{[k]}$ to represent $e_{i j}^{(k)} \otimes m_{i j}^{(k)}$. Note, however, that $m_{i j}^{[k]}$ is an element of $\mathbb{A}$, while $m_{i j}^{(k)}$ lies in $\mathbb{B}_{k}$. 
Consider $m \in \mathfrak{L}$, so that $m=\left[m_{i j}^{(k)}\right]$, where $m_{i j}^{[k]}=e_{i i}^{[k]} m e_{j j}^{[k]}$. Since $m \in \mathcal{Q}$, then

$$
m-\pi_{k}(m)=\left[\begin{array}{ccccc}
0 & m_{12}^{(k)} & m_{13}^{(k)} & \ldots & m_{1 p_{k}}^{(k)} \\
0 & 0 & m_{23}^{(k)} & \ldots & m_{2 p_{k}}^{(k)} \\
0 & 0 & 0 & \ldots & m_{3 p_{k}}^{(k)} \\
\vdots & \vdots & & \ddots & \\
0 & 0 & 0 & \ldots & 0
\end{array}\right]
$$

Now, by Lemma 20 each $m_{i j}^{[k]} \in \mathfrak{L}$ for $i \neq j$. Since $\mathfrak{L}$ is a linear manifold, we conclude that $m-\pi_{k}(m) \in \mathfrak{L}$ for each $k \geq 1$. Since $\mathfrak{L}$ is closed by assumption,

$$
m-\pi(m)=\lim _{k \rightarrow \infty} m-\pi_{k}(m) \in \mathcal{L} .
$$

Step Two: Next we show that $\mathcal{K}$ is closed.

Suppose $y_{n}=x_{n}-\pi\left(x_{n}\right) \in \mathcal{K}$ for $n \geq 1$ and that $\lim y_{n}=y \in \mathcal{A}$. Since $\mathcal{K} \subseteq \mathfrak{L}$ and $\mathfrak{L}$ is closed, $y \in \mathfrak{L}$. Now

$$
\pi(y)=\lim _{n} \pi\left(y_{n}\right)=\lim _{n} \pi\left(x_{n}-\pi\left(x_{n}\right)\right)=\lim _{n} \pi\left(x_{n}\right)-\pi^{2}\left(x_{n}\right)=0 .
$$

In particular, therefore, $y \in \mathfrak{L}$ and $y=y-\pi(y)$, so $y \in \mathcal{K}$ and the latter is closed.

Step Three: Finally, we show that $\mathcal{K}$ is a left $\mathfrak{D}$-module. The proof that it is a right $\mathfrak{D}$-module is similar and is left to the reader.

Temporarily fix $r \geq 1$. We begin by showing that $\mathcal{K}$ is a left $\mathfrak{D}_{p_{r}}$-module. Suppose $d \in \mathfrak{D}_{p_{r}}$ and let $x \in \mathfrak{L}$. We claim that it suffices to show that $d x-\pi(d x) \in$ $\mathfrak{L}$. Indeed, if this is the case, then $[d x-\pi(d x)]-\pi[d x-\pi(d x)]=d x-\pi(d x) \in \mathcal{K}$. This in turn says that whenever $x-\pi(x) \in \mathcal{K}$, then $d(x-\pi(x))=d x-\pi(d x) \in \mathcal{K}$, showing that indeed, $\mathcal{K}$ is a left $\mathfrak{D}_{p_{r}}$-module.

Let $k \geq 1$ and consider $d x-\pi_{k}(d x)$. As before, we may consider $\mathbb{A} \simeq^{*} \mathbf{M}_{p_{k}} \otimes \mathbb{B}_{k}$, where $\mathbb{B}_{k} \simeq^{*} e_{11}^{[k]} \mathbb{A} e_{11}^{[k]}$. Letting $d_{i}^{[k]}=e_{i i}^{[k]} d e_{i i}^{[k]}$ and $x_{i j}^{[k]}=e_{i i}^{[k]} x e_{j j}^{[k]}$, we obtain that $d x-\pi_{k}(d x)=\left[d_{i}^{(k)} x_{i j}^{(k)}\right]_{1 \leq i<j \leq p_{k}}$. To see that this is indeed an element of $\mathfrak{L}$, fix $1 \leq t \leq p_{k}$. Then since $x \in \mathfrak{L}, d_{t}^{[k]} \in \mathcal{Q}$, and $\mathfrak{L}$ is a Lie ideal, we have $\left[d_{t}^{[k]}, x\right] \in \mathfrak{L}$. But $\left[d_{t}^{[k]}, x\right]=\sum_{j=t}^{p_{k}} d_{t}^{[k]} x_{t j}^{[k]}-\sum_{i=1}^{t} x_{i t}^{[k]} d_{t}^{[k]}$. If $k \geq r$, then $d_{t}^{[k]}=e_{t t}^{[k]} d e_{t t}^{[k]}$ is a scalar, so that

$$
\left[d_{t}^{[k]}, x\right]=\left[\begin{array}{ccccccc}
0 & \ldots & 0 & -d_{t}^{(k)} x_{1 t}^{(k)} & 0 & \ldots & 0 \\
\vdots & \ldots & \vdots & \vdots & \vdots & \ldots & \vdots \\
0 & \ldots & 0 & -d_{t}^{(k)} x_{(t-1) t}^{(k)} & 0 & \ldots & 0 \\
0 & \ldots & 0 & 0 & d_{t}^{(k)} x_{t(t+1)}^{(k)} & \ldots & d_{t}^{(k)} x_{t p_{k}}^{(k)} \\
0 & \ldots & 0 & 0 & 0 & \ldots & 0 \\
\vdots & \ldots & \vdots & \vdots & \vdots & \ldots & \vdots \\
0 & \ldots & 0 & 0 & 0 & \ldots & 0
\end{array}\right]
$$


Similarly, since $e_{t t}^{[k]} \in \mathcal{Q}$, we have $\left[e_{t t}^{[k]},\left[d_{t}^{[k]}, x\right]\right] \in \mathfrak{L}$, and

$$
\left[e_{t t}^{[k]},\left[d_{t}^{[k]}, x\right]\right]=\left[\begin{array}{ccccccc}
0 & \ldots & 0 & d_{t}^{(k)} x_{1 t}^{(k)} & 0 & \ldots & 0 \\
\vdots & \ldots & \vdots & \vdots & \vdots & \ldots & \vdots \\
0 & \ldots & 0 & d_{t}^{(k)} x_{(t-1) t}^{(k)} & 0 & \ldots & 0 \\
0 & \ldots & 0 & 0 & d_{t}^{(k)} x_{t(t+1)}^{(k)} & \ldots & d_{t}^{(k)} x_{t p_{k}}^{(k)} \\
0 & \ldots & 0 & 0 & 0 & \ldots & 0 \\
\vdots & \ldots & \vdots & \vdots & \vdots & \ldots & \vdots \\
0 & \ldots & 0 & 0 & 0 & \ldots & 0
\end{array}\right] .
$$

Thus if $c_{t}^{[k]}=\frac{1}{2}\left[d_{t}^{[k]}, x\right]+\frac{1}{2}\left[e_{t t}^{[k]},\left[d_{t}^{[k]}, x\right]\right]$, then $c_{t}^{[k]} \in \mathfrak{L}$, and $c_{t}^{[k]}=\sum_{j=(t+1)}^{p_{k}} d_{t}^{[k]} x_{t j}^{[k]}$. Since $\mathfrak{L}$ is a linear manifold, $d x-\pi_{k}(d x)=\sum_{t=1}^{p_{k}} c_{t}^{[k]} \in \mathfrak{L}$.

Finally, since $\mathfrak{L}$ is closed, $d x-\pi(d x)=\lim _{k} d x-\pi_{k}(d x) \in \mathfrak{L}$, which proves that $\mathcal{K}$ is a left $\mathfrak{D}_{p_{r}}$-module. More generally, if $d \in \mathfrak{D}$ and $y \in \mathcal{K}$, then $d y=\lim _{r} d_{r} y$, where $d_{r} \in \mathfrak{D}_{p_{r}}$. Since each $d_{r} y \in \mathcal{K}$ from above and $\mathcal{K}$ is closed, $d y \in \mathcal{K}$, showing that $\mathcal{K}$ is a left $\mathfrak{D}$-module.

We now show that $\mathcal{K}$ is even better than just a $\mathfrak{D}$-bimodule: it is a diagonal disjoint associative ideal in $\mathcal{Q}$, and will play the rôle for TUHF algebras that the ideal defined before Lemma 4 played for nest algebras.

Proposition 22. Let $\mathfrak{L}$ be a closed Lie ideal in the triangular UHF algebra $\mathcal{Q}=$ $\lim \left(Q_{n}, \varphi_{n}\right)$, and for each $n \geq 1$ let $\mathfrak{L}_{n}=\mathfrak{L} \cap \mathcal{Q}_{n}$. If $\mathcal{K}$ is the bimodule $\{x-\pi(x) \mid x \in$ $\overrightarrow{\mathfrak{L}\}}$, then

$$
\mathcal{K}=\overline{\operatorname{span}}\left\{e_{i j}^{[n]} \in \mathfrak{L}_{n} \mid i<j, n \in \mathbb{N}\right\},
$$

and $\mathcal{K}$ is an associative ideal of $\mathcal{Q}$.

Proof. Set $\mathfrak{J}=\overline{\operatorname{span}}\left\{e_{i j}^{[n]} \in \mathfrak{L}_{n} \mid i<j, n \in \mathbb{N}\right\}$. If $\mathcal{K}=(0)$, then no matrix unit $e_{i j}^{[n]}$ with $i<j$ belongs to $\mathfrak{L}$, and so $\mathfrak{J}=(0)$.

Now suppose $\mathcal{K}$ is nonzero. The previous proposition shows that $\mathcal{K}$ is inductive, and so $\mathcal{K} \cap \mathcal{Q}_{n}$ is nonzero for all $n \geq n_{0}$ for some $n_{0}$. Since $\mathcal{K} \cap \mathcal{Q}_{n} \subseteq \mathfrak{L} \cap \mathcal{Q}_{n}$, then $\mathfrak{L} \cap \mathcal{Q}_{n}$ is nonzero for all such $n$.

First we must show that $\mathfrak{L}_{n}$ is a Lie ideal in $\mathcal{Q}_{n}$ for all $n \geq n_{0}$. To this end, let $m \in \mathfrak{L}_{n}$ and $y \in \mathcal{Q}_{n}$. Since $m, y \in \mathcal{Q}_{n}$, then $[m, y] \in \mathcal{Q}_{n}$, and since $m \in \mathfrak{L}$, then $[m, y] \in \mathfrak{L}$. This implies that $[m, y] \in \mathfrak{L}_{n}=\mathfrak{L} \cap \mathcal{Q}_{n}$. Clearly $\mathfrak{L}_{n}$ is a subspace since both $\mathfrak{L}$ and $\mathcal{Q}_{n}$ are, and hence $\mathfrak{L}_{n}$ is a Lie ideal of $\mathcal{Q}_{n}$. For all $n \geq n_{0}$, let

$$
\mathfrak{J}_{n}=\overline{\operatorname{span}}\left\{e_{i j}^{[n]} \in \mathfrak{L}_{n} \mid i<j\right\} .
$$

By Lemma 20 (ii) and (iii), we see that $\mathfrak{J}_{n}$ is an ideal of $\mathcal{Q}_{n}$. Clearly, $\bigcup_{n=n_{0}}^{\infty} \mathfrak{J}_{n}$ is dense in $\mathfrak{J}$.

We claim that $\mathfrak{J}_{n+1} \cap \mathcal{Q}_{n}=\mathfrak{J}_{n}$. Indeed, if $x \in \mathfrak{J}_{n+1} \cap \mathcal{Q}_{n}$, then we can write

$$
x=\sum_{\substack{i, j=1 \\ i \leq j}}^{p_{n}} x_{i j}(n) e_{i j}^{[n]}=\sum_{\substack{i, j=1 \\ i \leq j}}^{p_{n+1}} x_{i j}(n+1) e_{i j}^{[n+1]}
$$

for some $x_{i j}(n)$ and $x_{i j}(n+1) \in \mathbb{C}$. If $i<j$ and $x_{i j}(n) \neq 0$, then, since $x \in \mathfrak{L} \cap \mathcal{Q}_{n}$ $=\mathfrak{L}_{n}$ and $\mathfrak{L}_{n}$ is a Lie ideal of $\mathcal{Q}_{n}$, by Lemma 19 we have $x_{i j}(n) e_{i j}^{[n]} \in \mathfrak{L}$. Hence, 
$e_{i j}^{[n]} \in \mathfrak{L}_{n}$. Thus, $i<j$ implies that $e_{i j}^{[n]} \in \mathfrak{J}_{n}$. Now if $x_{i_{0} i_{0}}(n) \neq 0$ for some $i_{0}$, then since our maps are unital, there exists some $k_{0}$ so that $x_{k_{0} k_{0}}(n+1) \neq 0$. But this contradicts the fact that $x \in \mathfrak{J}_{n+1}$, and so we have $x_{i i}(n)=0$ for all $i$. This shows that $x \in \mathfrak{J}_{n}$, and so $\mathfrak{J}_{n+1} \cap \mathcal{Q}_{n}=\mathfrak{J}_{n}$ for all $n \geq n_{0}$. Hence, by [12, Proposition 2.5], $\mathfrak{J}$ is an associative ideal of $\mathcal{Q}$, so that $\mathfrak{J} \cap \mathcal{Q}_{n}=\mathfrak{J}_{n}$ for all $n$. A simple argument using the inductivity of $\mathcal{K}$ shows that $\mathfrak{J}=\mathcal{K}$, completing the proof.

Corollary 23. Let $\mathfrak{L}$ be a closed Lie ideal in $\mathcal{Q}$. Then $\mathcal{K}=\{x-\pi(x) \mid x \in \mathfrak{L}\}$ is a closed associative ideal of $\mathcal{Q}$ such that $\mathcal{K} \subseteq \mathfrak{L} \subseteq \mathcal{K}+\mathfrak{D}$.

Proof. Proposition 22 shows that $\mathcal{K}$ is an associative ideal of $\mathcal{Q}$, and Proposition 21 shows $\mathcal{K}$ is closed and $\mathcal{K} \subseteq \mathfrak{L}$. If $m \in \mathfrak{L}$, then $m=m-\pi(m)+\pi(m) \in \mathcal{K}+\mathfrak{D}$, and so we are done.

The above corollary shows that, as in the nest case, to every closed Lie ideal $\mathfrak{L}$ of $\mathcal{Q}$ there corresponds a closed associative ideal $\mathcal{K}_{\mathfrak{L}}$. We now show that associated to an arbitrary closed, associative, diagonal-disjoint ideal $\mathcal{K}$ of $\mathcal{Q}$ is a closed subspace $\mathfrak{D}_{\mathcal{K}}$ of the diagonal $\mathfrak{D}$ which is maximal with respect to the condition that $\mathfrak{L}(\mathcal{K})=$ $\mathfrak{D}_{\mathcal{K}}+\mathcal{K}$ defines a closed Lie ideal of $\mathcal{Q}$ whose diagonal-disjoint ideal $\mathcal{K}_{\mathfrak{L}(\mathcal{K})}$ is $\mathcal{K}$. Again, our choice of notation suggests a close connection between $\mathfrak{D}_{\mathcal{K}}$ and the algebra described in the definition preceding Proposition 9. However, the definition of $\mathfrak{D}_{\mathcal{K}}$ in the present case differs significantly from the nest algebra definition. Indeed, here $\mathfrak{D}_{\mathcal{K}}$ coincides with the intersection of $\mathfrak{D}$ with the lifting of the centre of $\mathcal{Q} / \mathcal{K}$.

If $\mathcal{Q}$ is a triangular UHF algebra, let $\mathcal{Q}_{s}$ be the maximal diagonal-disjoint ideal of $\mathcal{Q}$, i.e., the strong radical of $\mathcal{Q}$. Clearly we have $\mathcal{Q}_{s}=\overline{\operatorname{span}}\left\{e_{i j}^{[n]} \in \mathcal{Q} \mid i<j, n \in \mathbb{N}\right\}$.

Definition. Given an ideal $\mathcal{K}$ of $\mathcal{Q}$, define

$$
\mathfrak{D}_{\mathcal{K}}=\left\{d \in \mathfrak{D} \mid[d, q] \in \mathcal{K} \text { for all } q \in \mathcal{Q}_{s}\right\} .
$$

Proposition 24. Let $\mathcal{Q}$ be a triangular UHF algebra. If $\mathcal{K}$ is a closed diagonaldisjoint ideal of $\mathcal{Q}$, then $\mathfrak{D}_{\mathcal{K}}$ is an abelian $C^{*}$-algebra. Moreover, $\mathfrak{D}_{\mathcal{K}}$ is maximal in the sense that if $\mathcal{P}$ is a closed subspace of $\mathfrak{D}$ so that $\mathcal{P}+\mathcal{K}$ is a Lie ideal of $\mathcal{Q}$, then $\mathcal{P} \subseteq \mathfrak{D}_{\mathcal{K}}$.

Proof. Since $\mathfrak{D}_{\mathcal{K}} \subseteq \mathfrak{D}$ and both $\mathfrak{D}$ and $\mathcal{K}$ are closed, $\mathfrak{D}_{\mathcal{K}}$ is abelian and closed. If $\lambda \in \mathbb{C}$ and $a, b \in \mathfrak{D}_{\mathcal{K}}$, then we have $[\lambda a+b, q]=\lambda[a, q]+[b, q] \in \mathcal{K}$ and

$$
[a b, q]=a b q-q a b=a(b q-q b)+a q b-q a b=a[b, q]+[q, a] b \in \mathcal{K}
$$

for any $q \in \mathcal{Q}_{s}$. This shows that $\mathfrak{D}_{\mathcal{K}}$ is a closed abelian subalgebra of $\mathfrak{D}$.

It remains to show that $\mathfrak{D}_{\mathcal{K}}$ is self-adjoint. Suppose that $d \in \mathfrak{D}_{\mathcal{K}}$, and as in Step Three of Proposition 21, fix $n$ and write $d=\operatorname{diag}\left(d_{11}^{(n)}, d_{22}^{(n)}, \ldots, d_{p_{n} p_{n}}^{(n)}\right)$, where each $d_{k}^{[n]}=e_{k k}^{[n]} d e_{k k}^{[n]}$. If $i<j$, then

$$
\left[d, e_{i j}^{[n]}\right]=d_{i i}^{[n]} e_{i j}^{[n]}-e_{i j}^{[n]} d_{j j}^{[n]}=e_{i j}^{(n)} \otimes\left(d_{i i}^{(n)}-d_{j j}^{(n)}\right) \in \mathcal{K},
$$

since $d \in \mathfrak{D}_{\mathcal{K}}$. To show that $d^{*}$ is in $\mathfrak{D}_{\mathcal{K}}$, we must prove that $\left[d^{*}, e_{i j}^{[n]}\right]$ is in $\mathcal{K}$. But

$$
d^{*}=\operatorname{diag}\left(d_{11}^{(n)^{*}}, d_{22}^{(n)^{*}}, \ldots, d_{p_{n} p_{n}}^{(n)}{ }^{*}\right),
$$

and so $\left[d^{*}, e_{i j}^{[n]}\right]=e_{i j}^{(n)} \otimes\left(d_{i i}^{(n)^{*}}-d_{j j}^{(n)^{*}}\right)$. Thus it suffices to prove that

$$
e_{i j}^{(n)} \otimes\left(d_{i i}^{(n)^{*}}-d_{j j}^{(n)^{*}}\right) \in \mathcal{K} .
$$


Let $\mathbb{A}$ be the UHF algebra corresponding to $\mathcal{Q}$. As in Proposition 21, we view $\mathbb{A}$ as $\mathbf{M}_{p_{n}} \otimes \mathbb{B}_{n}$. Notice that since $d \in \mathfrak{D}_{\mathcal{K}} \subseteq \mathfrak{D}$, then $\left(d_{i i}^{(n)}-d_{j j}^{(n)}\right) \in \mathfrak{D}_{\mathbb{B}_{n}}$, where $\mathfrak{D}_{\mathbb{B}_{n}}$ is the diagonal of $\mathbb{B}_{n}$.

Recall that $i<j$ are fixed. Given $k \in \mathcal{K}$, define $k_{i j}^{[n]}=e_{i i}^{[n]} k e_{j j}^{[n]} \in \mathcal{K}$. Thus, $k_{i j}^{[n]}=e_{i j}^{(n)} \otimes k_{i j}^{(n)}$, where $k_{i j}^{(n)}$ belongs to $\mathbb{B}_{n}$. Define

$$
\mathfrak{J}_{\mathbb{B}_{n}}=\left\{r \in \mathfrak{D}_{\mathbb{B}_{n}} \mid e_{i j}^{(n)} \otimes r \in \mathcal{K}\right\} .
$$

We now show that $\mathfrak{J}_{\mathbb{B}_{n}}$ is an ideal of $\mathfrak{D}_{\mathbb{B}_{n}}$.

Since $\mathcal{K}$ is a closed subspace of $\mathcal{Q}$, it follows that $\mathfrak{J}_{\mathbb{B}_{n}}$ is a closed subspace of $\mathfrak{D}_{\mathbb{B}_{n}}$. In addition, if $b \in \mathfrak{D}_{\mathbb{B}_{n}}$ and $r \in \mathfrak{J}_{\mathbb{B}_{n}}$, then $e_{i i}^{(n)} \otimes b \in e_{i i}^{[n]} \mathcal{Q} e_{i i}^{[n]} \subseteq \mathcal{Q}$ and $e_{i j}^{(n)} \otimes r \in \mathcal{K}$. Thus, $\left(e_{i i}^{(n)} \otimes b\right)\left(e_{i j}^{(n)} \otimes r\right)=e_{i j}^{(n)} \otimes b r \in \mathcal{K}$. Since $b r \in \mathbf{D}_{\mathbb{B}_{n}}$, then, by definition, $b r \in \mathfrak{J}_{\mathbb{B}_{n}}$. This shows that $\mathfrak{J}_{\mathbb{B}_{n}}$ is a left ideal of $\mathfrak{D}_{\mathbb{B}_{n}}$. Similarly, $\mathfrak{J}_{\mathbb{B}_{n}}$ is a right ideal. Thus, $\mathfrak{J}_{\mathbb{B}_{n}}$ is a closed ideal in the $\mathrm{C}^{*}$-algebra $\mathbf{D}_{\mathbb{B}_{n}}$, and so it is self-adjoint.

Since $\left(d_{i i}^{(n)}-d_{j j}^{(n)}\right) \in \mathfrak{D}_{\mathbb{B}_{n}}$ and since $\left[d, e_{i j}^{[n]}\right] \in \mathcal{K}$ if and only if $e_{i j}^{(n)} \otimes\left(d_{i i}^{(n)}-d_{j j}^{(n)}\right) \in$ $\mathcal{K}$, then by the definition of $\mathfrak{J}_{\mathbb{B}_{n}}$ we have $\left(d_{i i}^{(n)}-d_{j j}^{(n)}\right) \in \mathfrak{J}_{\mathbb{B}_{n}}$. It follows that $\left(d_{i i}^{(n)^{*}}-d_{j j}^{(n)^{*}}\right) \in \mathfrak{J}_{\mathbb{B}_{n}}$, and therefore $e_{i j}^{(n)} \otimes\left(d_{i i}^{(n)^{*}}-d_{j j}^{(n)^{*}}\right)=\left[d^{*}, e_{i j}^{[n]}\right] \in \mathcal{K}$.

Since $i<j$ was arbitrary, this shows that $d^{*} \in \mathfrak{D}_{\mathcal{K}}$, and so $\mathfrak{D}_{\mathcal{K}}$ is self-adjoint, and hence, a $\mathrm{C}^{*}$-algebra.

Finally, to see the second statement, suppose that $\mathcal{P}$ is a closed subspace of $\mathfrak{D}$ so that $\mathcal{P}+\mathcal{K}$ is a closed Lie ideal. Clearly $\pi(\mathcal{P}+\mathcal{K})=\mathcal{P}$ and $\mathcal{K}=\{x \in \mathcal{P}+\mathcal{K} \mid x \in$ $\operatorname{ker}(\pi)\}$. Let $m \in \mathcal{P}$. Since $\mathcal{P}+\mathcal{K}$ is a Lie ideal, we have $[m, q] \in \mathcal{P}+\mathcal{K}$ for all $q \in \mathcal{Q}_{s}$. But $\pi([m, q])=\pi(m q-q m)=m \pi(q)-\pi(q) m=0$, so that $[m, q] \in \mathcal{K}$. In other words, $m \in \mathfrak{D}_{\mathcal{K}}$, and hence $\mathcal{P} \subseteq \mathfrak{D}_{\mathcal{K}}$.

The next result is our main theorem in the triangular UHF algebra case.

Theorem 25. Let $\mathcal{Q}$ be a triangular UHF algebra and let $\mathfrak{L}$ be a closed Lie ideal of $\mathcal{Q}$. There exist a closed, diagonal-disjoint, associative ideal $\mathcal{K}$ and a closed, abelian $C^{*}$-subalgebra $\mathfrak{D}_{\mathcal{K}}$ of the diagonal $\mathfrak{D}$ such that

$$
\mathcal{K} \subseteq \mathfrak{L} \subseteq \mathfrak{D}_{\mathcal{K}}+\mathcal{K}
$$

Moreover, if $\mathfrak{M}$ is any closed subspace of $\mathfrak{D}_{\mathcal{K}}$, then $\mathfrak{L}(\mathfrak{M}):=\mathfrak{M}+\mathcal{K}$ defines a closed Lie ideal of $\mathcal{Q}$ whose corresponding closed, diagonal-disjoint, associative ideal is $\mathcal{K}$ as well.

Proof. The first statement is a summary of the above theorems. The second is straightforward, since $\mathfrak{M}+\mathcal{K}$ is contained in the lifting of the centre of $\mathcal{Q} / \mathcal{K}$.

It remains only to obtain a better description of the diagonal part $\mathfrak{D}_{\mathcal{K}}$ associated with a given closed, diagonal-disjoint, associative ideal $\mathcal{K}$. The following proposition does exactly this for an important class of triangular UHF algebras in the case where the algebra $\mathfrak{D}_{\mathcal{K}}$ is known to be inductive. It is an open question to decide whether for all closed ideals $\mathcal{K}$, the corresponding $\mathfrak{D}_{\mathcal{K}}$ must be inductive.

A triangular UHF algebra is called a full nest algebra if it has a presentation $\underset{\lim }{\longrightarrow}\left(\mathcal{Q}_{n}, \varphi_{n}\right)$ such that each embedding $\varphi_{n}$ is a nest embedding, i.e., if for every $n$, $\overrightarrow{\varphi_{n}}\left(\right.$ Lat $\left.\mathcal{Q}_{n}\right) \subseteq$ Lat $\mathcal{Q}_{n+1}$, where Lat $\mathcal{B}$ denotes the lattice of invariant projections of $\mathcal{B}$. See [7] for more details on full nest algebras. 
Proposition 26. Let $\mathcal{Q}$ be a full nest algebra, $\mathfrak{L}$ a closed Lie ideal in $\mathcal{Q}$, and $\mathcal{K}$ the associative ideal $\{x-\pi(x) \mid x \in \mathfrak{L}\}$. If $\mathfrak{D}_{\mathcal{K}}$ is inductive, then

$$
\mathfrak{D}_{\mathcal{K}}=\overline{\operatorname{span}}\left\{p \mid p \in \text { Lat } \mathcal{Q}, p \mathcal{Q} p^{\perp} \subseteq \mathcal{K}\right\} .
$$

Proof. If $\mathcal{P}_{\mathcal{K}}=\overline{\operatorname{span}}\left\{p \mid p \in\right.$ Lat $\left.\mathcal{Q}, p \mathcal{Q} p^{\perp} \subseteq \mathcal{K}\right\}$, then clearly $\mathcal{P}_{\mathcal{K}}$ is a closed subspace of $\mathfrak{D}$. To see that $\mathfrak{D}_{\mathcal{K}} \subseteq \mathcal{P}_{\mathcal{K}}$, we first show that for any $n \geq 1, \mathfrak{D}_{\mathcal{K}} \cap \mathcal{Q}_{n} \subseteq \mathcal{P}_{\mathcal{K}}$.

To this end, assume that $d \in \mathfrak{D} \cap \mathcal{Q}_{n}$ and $d \notin \mathcal{P}_{\mathcal{K}}$. Observe that Lat $\mathcal{Q}_{k}=\left\{q_{m}^{[k]}=\right.$ $\left.\sum_{i=1}^{m} e_{i i}^{[k]} \mid 1 \leq m \leq p_{k}\right\}$, where $p_{k}$ is the dimension of $\mathcal{Q}_{k}$, and, moreover, since $\mathcal{Q}$ is a full nest algebra, Lat $\mathcal{Q}=\bigcup_{k}$ Lat $\mathcal{Q}_{k}$. Since $d \notin \mathcal{P}_{\mathcal{K}}$, we have that $d \neq 0$, $d \neq \lambda I$ for $\lambda \in \mathbb{C}$, and

$$
d \notin \operatorname{span}\left\{q \mid q \in \text { Lat } \mathcal{Q}_{n}, q \mathcal{Q} q^{\perp} \subseteq \mathcal{K}\right\},
$$

for any $n$.

Since $d \in \mathfrak{D} \cap \mathcal{Q}_{n}$, we can write $d=\sum_{i=1}^{p_{n}} d_{i} e_{i i}^{[n]}$ for $d_{i} \in \mathbb{C}$. Observe that

$$
d=d_{p_{n}} q_{p_{n}}^{[n]}+\left(d_{p_{n}-1}-d_{p_{n}}\right) q_{p_{n}-1}^{[n]}+\cdots+\left(d_{2}-d_{3}\right) q_{2}^{[n]}+\left(d_{1}-d_{2}\right) q_{1}^{[n]} .
$$

Provided $\mathcal{K}$ is nonzero, (3) ensures there is some $1 \leq l \leq r-1$ so that $d_{l} \neq d_{l+1}$ and $q_{l} \mathcal{Q} q_{l}^{\perp} \nsubseteq \mathbb{K}$. Now by [8, Lemma 2.3], $q_{l} \mathcal{Q} q_{l}^{\perp}$ is generated by a sequence of matrix units $\left\{f_{k}\right\}_{k \geq n}$, where $f_{k}=e_{l_{k}, l_{k}+1}^{[k]}$ and $l_{k}$ is the position of the largest restriction of $e_{l l}^{[n]}$ in $\mathcal{Q}_{k}$. Since $q_{l} \mathcal{Q} q_{l}^{\perp} \nsubseteq \mathcal{K}$, then for some $k \geq n, f_{k} \notin \mathcal{K}$. A direct calculation shows that

$$
\left[d, f_{k}\right]=\left[\varphi_{k, n}(d), f_{k}\right]=\left(d_{l}-d_{l+1}\right) f_{k} .
$$

Since $d_{l} \neq d_{l+1}$ and $f_{k} \notin \mathcal{K}$, then we have found an element of $\mathcal{Q}_{s}$, namely $f_{k}$, so that $\left[d, f_{k}\right] \notin \mathcal{K}$. Hence, $d \notin \mathfrak{D}_{\mathcal{K}}$.

This shows that $\mathfrak{D}_{\mathcal{K}} \cap \mathcal{Q}_{n} \subseteq \mathcal{P}_{\mathcal{K}} \cap \mathcal{Q}_{n}$ for all $n \geq 1$. Since by hypothesis $\mathfrak{D}_{\mathcal{K}}$ is inductive, it follows that $\mathfrak{D}_{\mathcal{K}} \subseteq \mathcal{P}_{\mathcal{K}}$.

To prove the reverse containment, first assume that $\mathcal{P}_{\mathcal{K}}$ is trivial, i.e., $\mathcal{P}_{\mathcal{K}}=$ $\{0, I\}$. Then by the first part of the proof, we have that $\mathfrak{D}_{\mathcal{K}} \cap \mathcal{Q}_{n}$ is trivial for all $n$, and so by inductivity $\mathfrak{D}_{\mathcal{K}}$ is trivial.

Now let $q$ be a nontrivial invariant projection for $\mathcal{Q}$ so that $q \mathcal{Q} q^{\perp} \subseteq \mathcal{K}$. Also, let $e$ be a matrix unit in $\mathcal{Q}_{s}$, i.e., an off-diagonal matrix unit. Since $q \in$ Lat $\mathcal{Q}$, then $e$ must belong to one of $q \mathcal{Q} q, q^{\perp} \mathcal{Q} q^{\perp}$, or $q \mathcal{Q} q^{\perp}$. In either of the first two cases, it is immediate that $q e-e q=0$, so $[q, e] \in \mathcal{K}$. If $e \in q \mathcal{Q} q^{\perp}$, then $q e=e$ and $e q=\left(e q^{\perp}\right) q=0$, so $[q, e]=e \in q \mathcal{Q} q^{\perp} \subseteq \mathcal{K}$. Thus, we have shown that for any matrix unit $e$ in $\mathcal{Q}_{s},[q, e] \in \mathcal{K}$. It follows that $[q, d] \in \mathcal{K}$ for all $d \in \mathcal{Q}_{s}$, and so $q$ lies in $\mathfrak{D}_{\mathcal{K}}$. Since $\mathfrak{D}_{\mathcal{K}}$ is a closed subspace of $\mathfrak{D}$, the proof is complete.

\section{NON-ClOSED LIE IDEALS IN TRIANGULAR UHF ALGEBRAS}

In the proof of Step One of Proposition 21, we described the canonical projection $\pi$ of the UHF algebra $\mathbb{A}$ onto its canonical masa $\mathfrak{D}=\mathcal{Q} \cap \mathcal{Q}^{*}$. Note that $\left.\pi\right|_{\mathcal{Q}}$ is in fact a homomorphism, which should not be too surprising since it parallels the result for nest algebras. Indeed, consider $a, b \in \mathcal{Q}$ and the map

$$
\begin{aligned}
& \pi_{k}: \mathbb{A} \longrightarrow \mathfrak{D}, \\
& a \longmapsto \sum_{i} e_{i i}^{[k]} a e_{i i}^{[k]} .
\end{aligned}
$$


Since $\pi_{k}(a b)=\sum_{i} e_{i i}^{[k]} a b e_{i i}^{[k]}, a=\sum_{r \leq t} e_{r r}^{[k]} a e_{t t}^{[k]}$, and $b=\sum_{s \leq v} e_{s s}^{[k]} b e_{v v}^{[k]}$, we see that

$$
\begin{aligned}
\pi_{k}(a b) & =\sum_{i} \sum_{r \leq t} \sum_{s \leq v} e_{i i}^{[k]}\left(e_{r r}^{[k]} a e_{t t}^{[k]}\right)\left(e_{s s}^{[k]} b e_{v v}^{[k]}\right) e_{i i}^{[k]} \\
& =\sum_{i} e_{i i}^{[k]} a e_{i i}^{[k]} b e_{i i}^{[k]} \\
& =\sum_{i} e_{i i}^{[k]} a e_{i i}^{[k]} \sum_{j} e_{j j}^{[k]} b e_{j j}^{[k]} \\
& =\pi_{k}(a) \pi_{k}(b) .
\end{aligned}
$$

But this means that $\pi(a b)=\lim _{k} \pi_{k}(a b)=\lim _{k} \pi_{k}(a) \pi_{k}(b)=\pi(a) \pi(b)$, and so $\left.\pi\right|_{\mathcal{Q}}$ is a homomorphism, as claimed. In particular, therefore, if $a=a^{2} \in \mathcal{Q}$, then $\pi(a)=\pi\left(a^{2}\right)=\pi(a)^{2}$, and so $\pi(a)$ is an idempotent in $\mathfrak{D}$.

It is well-known that if $\mathcal{Q}$ is a unital associative algebra and $E$ denotes the (nonclosed) linear span of the idempotents in $\mathcal{Q}$, then $E$ is a Lie ideal of $\mathcal{Q}$ [6]. Our present goal is to show that in the case where $\mathcal{Q}$ is a triangular UHF algebra as above, $E$ is not closed. (In contrast, note that in $\mathcal{B}(\mathcal{H})$, every element is the span of at most 8 idempotents. On the other hand, the set of compact operators is an AF algebra for which $E$ consists of the finite rank operators. At the other end of the spectrum, $\mathcal{C}([0,1])$ has only the trivial idempotents, whose span is nowhere dense. Although the linear span of the idempotents in a UHF algebra $\mathbb{A}$ is dense, it is not known whether or not it is closed, and hence all of $\mathbb{A}$.)

Now if $a \in \mathcal{Q}$ is idempotent, then $\sigma(a) \in\{0,1\}$. So if $q=\lambda_{1} a_{1}+\lambda_{2} a_{2}+$ $\ldots+\lambda_{n} a_{n}$ is a linear combination of idempotents, then $\pi(q)=\sum_{i=1}^{n} \lambda_{i} \pi\left(a_{i}\right)$ is a linear combination of idempotents in the commutative Banach algebra $\mathfrak{D}$. But then $\sigma(\pi(q)) \subseteq \sum_{i=1}^{n} \lambda_{i} \sigma\left(\pi\left(a_{i}\right)\right)$, and hence it has finite cardinality. Clearly $\mathfrak{D}$ contains many elements with infinite spectrum, and thus $\mathfrak{D}$ is not spanned by its idempotents. We conclude that neither is $\mathcal{Q}$, and therefore $E$ is an example of a non-closed Lie ideal of $\mathcal{Q}$.

\section{REFERENCES}

1. K.R. Davidson, Nest algebras, Pitman Research Notes in Mathematics, no. 191, Longman Sci. Tech., Harlow, 1988. MR 90f: 47062

2. J.A. Erdos and S.C. Power, Weakly closed ideals of nest algebras, J. Operator Theory 7 (1982), 219-235. MR 84a:47056

3. C.K. Fong, C.R. Miers, and A.R. Sourour, Lie and Jordan ideals of operators on Hilbert space, Proc. Amer. Math. Soc. 84 (1982), 516-520. MR 84g:47039

4. K.-H. Förster and B. Nagy, Lie and Jordan ideals in $B c_{0}$ and $B\left(\ell^{\rho}\right)$, Proc. Amer. Math. Soc. 117 (1993), 673-677. MR 93d:47086

5. J. Glimm, On a certain class of operator algebras, Trans. Amer. Math. Soc. 95 (1960), 318-340. MR 22:2915

6. I.N. Herstein, Topics in ring theory, University of Chicago Press, Chicago, 1969. MR 42:6018

7. A. Hopenwasser and J.R. Peters, Full nest algebras, Illinois J. Math. 38 (1994), 501-520. MR 95c: 47047

8. T.D. Hudson, Ideals in triangular AF algebras, Proc. London Math. Soc. (3) 69 (1994), 345-376. MR 95d:46060

9. L. Marcoux, On the closed Lie ideals of certain $\mathrm{C}^{*}$-algebras, Integral Equations Operator Theory 22 (1995), 463-475. MR 96f:46123

10. C.R. Miers, Closed Lie ideals in operator algebras, Canad. J. Math. 33 (1981), 1271-1278. MR 82m: 46064

11. G.J. Murphy, Lie ideals in associative algebras, Canad. Math. Bull. 27 (1984), 10-15. MR 85b:16030 
12. J.R. Peters, Y.T. Poon, and B.H. Wagner, Triangular AF algebras, J. Operator Theory 23 (1990), 81-114. MR 91h:46102

13. _ Analytic TAF algebras, Canad. J. Math. 45 (1993), 1009-1031, Correction 46 (1994), 395-396. MR 94m:46113a,b

14. S.C. Power, Limit algebras, Pitman Research Notes in Mathematics, no. 278, Longman Sci. Tech., Harlow, 1992. MR 94g:46001

Department of Mathematics, East Carolina University, Greenville, North Carolina, $27858-4353$

E-mail address: tdh@math.ecu.edu

Department of Mathematical Sciences, University of Alberta, Edmonton, Alberta, Canada T6G 2G1

E-mail address: L.Marcoux@ualberta.ca

Department of Mathematics, University of Victoria, Victoria, British Columbia, Canada V8W 3P4

E-mail address: sourour@math.uvic.ca 